SC-R-66-896

Technical Report

A pril 1966

\title{
TABLES OF $\Psi$-FUNCTIONS
}

Charles W. Harrison, Jr. *

Ronold W. P. King $†$

*Sandia Corporation, Albuquerque

tConsultant to the Sandia Corporation and

Professor of Applied Physics, Harvard University

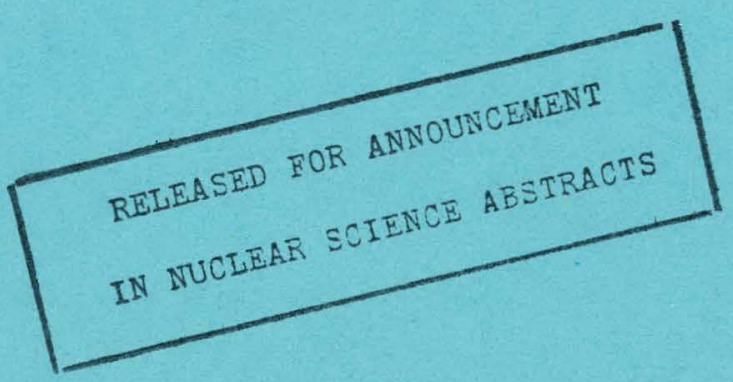

SANDIA CORPORATION

PRIME CONTRACTOR TO THE U.S. ATOMIC ENERGY COMMISSION | ALBUQUERQUE, NEW MEXICO; LIVERMORE, CALIFORNIA; TONOPAH, NEVADA 


\section{DISCLAIMER}

This report was prepared as an account of work sponsored by an agency of the United States Government. Neither the United States Government nor any agency Thereof, nor any of their employees, makes any warranty, express or implied, or assumes any legal liability or responsibility for the accuracy, completeness, or usefulness of any information, apparatus, product, or process disclosed, or represents that its use would not infringe privately owned rights. Reference herein to any specific commercial product, process, or service by trade name, trademark, manufacturer, or otherwise does not necessarily constitute or imply its endorsement, recommendation, or favoring by the United States Government or any agency thereof. The views and opinions of authors expressed herein do not necessarily state or reflect those of the United States Government or any agency thereof. 


\section{DISCLAIMER}

Portions of this document may be illegible in electronic image products. Images are produced from the best available original document. 


\section{Issued by Sandia Corporation, a prime contractor to the United States Atomic Energy Commission}

\section{LEGAL NOTICE}

This report was prepared as an account of Government sponsored work. Neither the United States, nor the Commission, nor any person acting on behalf of the Commission:

A. Makes any warranty or representation, expressed or implied, with respect to the accuracy, completeness, or usefulness of the information contained spect to the accuracy, completeness, or usefulness of the information contained
in this report, or that the use of any information, apparatus, method, or process disclosed in this report may not infringe privately owned rights; or

B. Assumes any liabilities with respect to the use of, or for damages resulting from the use of any information, apparatus, method, or process disclosed in this report.

As used in the above, "person acting on behalf of the Commission" includes any employee or contractor of the Commission, or employee of such contractor. to the extent that such employee or contractor of the Commission, or employee of such contractor prepares, disseminates, or provides access to, any information pursuant to his employment or contract with the Commission, or his emptoyment pursuant to his employment or contract with the Commission, or his eiriployme with such contractor. 
H.c. $1.00: 50$

\author{
$\mathrm{SC}-\mathrm{R}-66-896$
}

\title{
TABLES OF $\Psi$-FUNCTIONS
}

\author{
Charles W. Harrison, Jr. * \\ Ronold W. P. Kingt \\ *Sandia Corporation, Albuquerque \\ $t_{\text {Consultant to the Sandia Corporation and }}$ \\ Professor of Applied Physics, Harvard University
}

April 1966

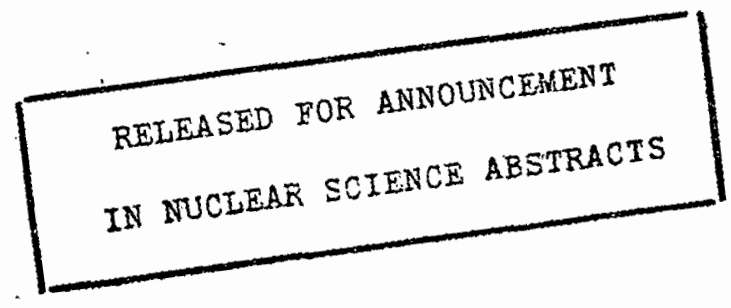

\section{SUMMARY}

Certain integrals useful in the approximate solution of the integral equation for the current in a cylindrical antenna are evaluated and represented graphically and in tabular form.

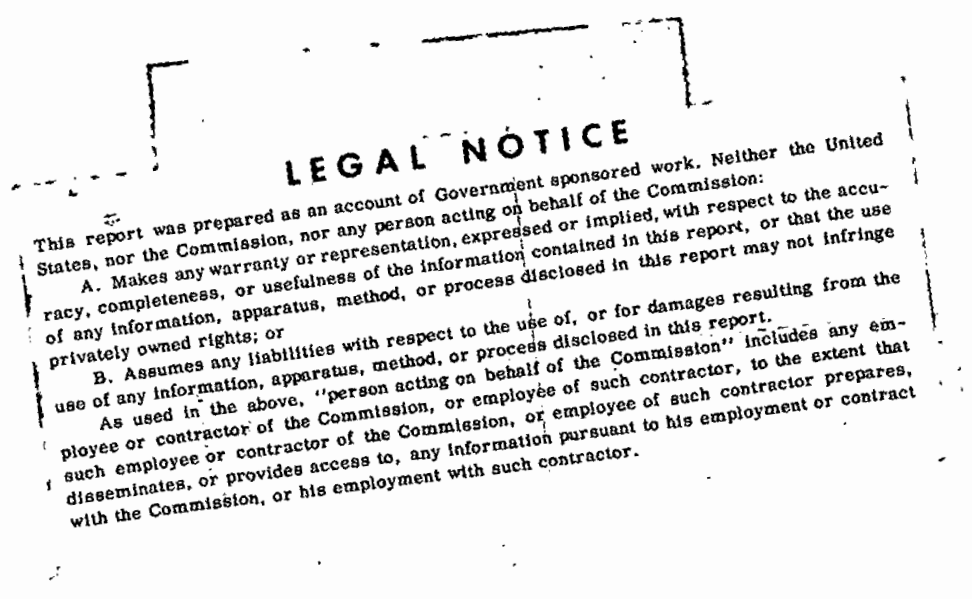




\section{ACKNOW LEDGMENT}

E. A. Aronson programmed the Sandia Laboratory CDC-3600 computer for calculating the $\Psi$-functions. The manuscript was prepared by Deyoe S.tark. 
The vector potential due to a current $I(z)$ in a thin cylindrical antenna of radius a and length $2 \mathrm{~h}$ is given by

$$
A(z)=\frac{\mu_{0}}{4 \pi} \int_{-h}^{h} I\left(z^{\prime}\right) \frac{e^{-j k} o_{o}^{r}}{r} d z^{\prime}
$$

where

$$
r=\sqrt{\left(z-z^{\prime}\right)^{2}+a^{2}}
$$

is the distance from the element $\mathrm{dz}^{\prime}$ at $\mathrm{z}^{\prime}$ on the axis to the point $\mathrm{z}$ on the surface. The corresponding expression for the vector potential at the end of the antenna is given by (1) and (2) with $z=h$. The notation

$$
r_{h}=\sqrt{\left(h-z^{\prime}\right)^{2}+a^{2}}
$$

is used.

Approximate methods of solving the integral equation for the current in a cylindrical antenna make use of functions of the type

$$
\Psi(z)=\frac{4 \pi}{\mu_{0}} \frac{A(z)}{I(z)}=\frac{1}{I(z)} \int_{-h}^{h} I\left(z^{\prime}\right) \frac{e^{-j k} o^{r}}{r} d z^{\prime}
$$


These functions are frequently slowly varying as functions of $z$ (except near $z=h$ ) for antennas that are not too long.

As shown by King and $\mathrm{Wu}^{1}$ an excellent approximation of the current distribution in a cylindrical antenna has the form

$$
\begin{aligned}
I(z)= & C\left[\sin k_{o}(h-|z|)+T_{U}\left(\cos k_{o} z-\cos k_{o} h\right)\right. \\
& \left.+T_{D}\left(\cos \frac{1}{2} k_{o} z-\cos \frac{1}{2} k_{o} h\right)\right] .
\end{aligned}
$$

It follows that functions of the type (4) with $I\left(z^{\prime}\right)$ proportional to each of the three trigonometric functions in (5), viz., $\sin \mathrm{k}_{\mathrm{o}}(\mathrm{h}-|\mathrm{z}|),\left(\cos \mathrm{k}_{\mathrm{o}} \mathrm{z}-\cos \mathrm{k}_{\mathrm{o}} \mathrm{h}\right)$, and $\left(\cos \frac{1}{2} k_{o} z-\cos \frac{1}{2} k_{o} h\right)$ are of interest.

Owing to the fact that the part of (1) that involves the imaginary part of the kernel has quite different properties from the part determined by the real part of the kernel, the integral (4) with $I\left(z^{\prime}\right)$ given by $\sin \mathrm{k}_{\mathrm{o}}\left(\mathrm{h}-\left|\mathrm{z}^{\prime}\right|\right)$ ) or (cos $\mathrm{k}_{\mathrm{o}} \mathrm{z}^{\prime}$ $-\cos \mathrm{k}_{\mathrm{O}} \mathrm{h}$ ) in the numerator and with $\mathrm{I}(\mathrm{z}) \sim\left(\cos \frac{1}{2} \mathrm{k}_{\mathrm{o}} \mathrm{z}-\cos \frac{1}{2} \mathrm{k}_{\mathrm{o}} \mathrm{h}\right)$ in the denominator are also of interest.

Tables of the following integrals have been computed as functions of $\mathrm{z} / \mathrm{h}$ for $\mathrm{h} / \lambda=0.125,0.250,0.375,0.500,0.625,0.750,0.875$, and 1.000 with $\Omega=2 \ln \frac{2 h}{a}=8.54,9.92$, and 15 . Typical graphs of the corresponding real and imaginary parts are shown in Figures 1 through 4 for $\Omega=8.54$.

R. W. P. King and T. T. Wu, "Currents, Charges and Near Fields of Cylindrical Antennas," Radio Science, Vol. 69D, pp. 429-446 (March 1965). 


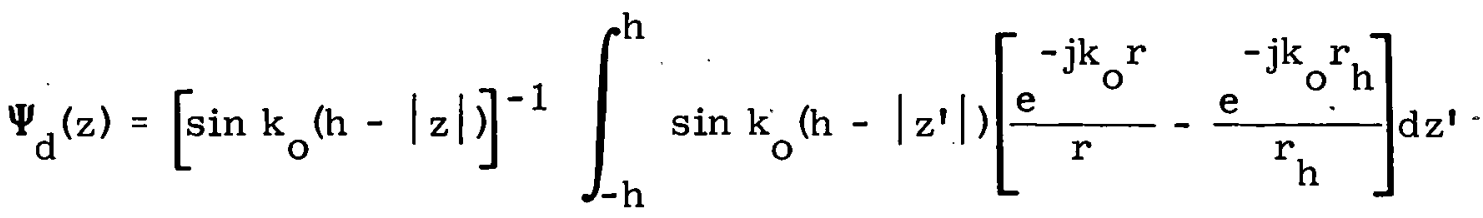

$$
\begin{aligned}
& \Psi_{d U}(z)=\left[\cos k_{o} z-\cos k_{o} h\right]^{-1} \int_{-h}^{h}\left[\cos k_{o} z^{\prime}-\cos k_{o} h\right]\left[\frac{e^{-j k}{ }_{o}^{r}}{r}-\frac{e^{-j k} r_{o} h}{r_{h}}\right] d z^{\prime} \\
& \Psi_{\mathrm{dD}}(\mathrm{z})=\left[\cos \frac{1}{2} \mathrm{k}_{\mathrm{o}} \mathrm{z}-\cos \frac{1}{2} \mathrm{k}_{\mathrm{o}} \mathrm{h}\right]^{-1} \int_{-\mathrm{h}}^{\mathrm{h}}\left[\cos \frac{1}{2} \mathrm{k}_{\mathrm{o}^{\prime}} \mathrm{z}^{\prime}-\cos \frac{1}{2} \mathrm{k}_{\mathrm{o}} \mathrm{h}\right] \\
& {\left[\frac{e^{-j k r} \cdot o}{r}-\frac{e^{-j k} o_{h}}{r_{h}}\right] d z^{\prime}} \\
& \Psi_{\mathrm{dI}}^{\prime}(\mathrm{z})=-\left[\cos \frac{1}{2} \mathrm{k}_{\mathrm{o}} \mathrm{z}-\cos \frac{1}{2} \mathrm{k}_{\mathrm{o}} \mathrm{h}\right]^{-1} \int_{-\mathrm{h}}^{\mathrm{h}} \sin \mathrm{k}_{\mathrm{o}}\left(\mathrm{h}-\left|\mathrm{z}^{\prime}\right|\right)\left[\frac{\sin \mathrm{k}_{\mathrm{o}} \mathrm{r}}{\mathrm{r}}-\frac{\sin \mathrm{k}_{\mathrm{o}} \mathrm{r}_{\mathrm{h}}}{\mathrm{r}_{\mathrm{h}}}\right] \mathrm{dz} \mathrm{z}^{\prime} \\
& \Psi_{\mathrm{dUI}}^{\prime}(\mathrm{z})=-\left[\cos \frac{1}{2} \mathrm{k}_{\mathrm{o}} \mathrm{z}-\cos \frac{1}{2} \mathrm{k}_{\mathrm{o}} \mathrm{h}\right]^{-1} \int_{-\mathrm{h}}^{\mathrm{h}}\left[\cos \mathrm{k}_{\mathrm{o}} \mathrm{z}^{\prime}-\cos \mathrm{k}_{\mathrm{o}} \mathrm{h}\right] \\
& {\left[\frac{\sin \mathrm{k}_{\mathrm{o}}^{\mathrm{r}}}{\mathrm{r}}-\frac{\sin \mathrm{k}_{\mathrm{o}} \mathrm{r}_{\mathrm{h}}}{\mathrm{r}_{\mathrm{h}}}\right] \mathrm{dz}} \\
& r=\sqrt{\left(z-z^{\prime}\right)^{2}+a^{2}}, \quad r_{h}=\sqrt{\left(h-z^{\prime}\right)^{2}+a^{2}}
\end{aligned}
$$

5-6 
$\Omega=8.54$

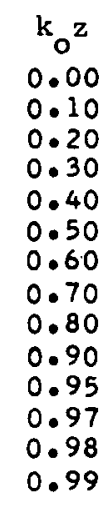

$\Psi_{d}(z)$

5.5092

5.7748

5.8911

5.9403

5.9405

5.8955

5.8001

5.6386

5.3728

4.8945
4.4677

4.4677
4.2234

4.2234
4.0790

3.9200

0.99

$-0.0800$

$-0.0862$

$-0.0523$

$-0.0982$

$-0.1041$

$-0.1100$

$-0.1217$

$-0.1276$

$-0.1336$

$-0.1366$

$-0.1378$

$-0.1390$
0.00

0.10

0.20

0.30

0.40

0.50

0.60

0.70

0.80
0.90

0.90
0.95

0.97

0.98

0.99

6.2264
6.2182
6.1928
6.1487
6.0825
5.9885
5.8567
5.6684
5.3838
4.8935
4.4631
4.2177
4.0729
3.9134

3.9134

$-0.6330$

$-0.6337$

$-0.6357$

$-0.6391$

$-0.6439$

$-0.6503$

$-0.6582$

$-0.6679$

$-0.6794$

$-0.6930$

$-0.7007$

$-0.7039$

$-0.7055$

$-0.7072$
$\Psi_{\mathrm{dU}}(\mathrm{z})$

5.8285

5.8192

5.7906

5.7411

5.6672

5.5631

5.4187

5.2153

4.9128

4.4021

3.9613

3.7122
3.5657

3.4050

$\Omega=8.54$

6.2264

6.2182

6.1928
6.1487

6.0825

5.9885

5.8567

5.6684

5.3838

4.8935

4.4631

4. 2177

4.0729

3.9134

$\Omega=8.54$

6.3233

6.3181

6.3022

6.2744

6.2322

6.1713

6.0837

5.9538

5.7461

5.3571

4.9876

4.9876

4.7677
4.6353

4.4878
$-0.0996$

$-0.0996$

$-0.0997$

$-0.0998$

$-0.0999$

$-0.1001$

$-0.1004$

$-0.1007$

$-0.1010$

$-0.1014$

$-0.1016$

$-0.1017$

$-0.1017$

$-0.1018$

$-0.6330$

$-0.6337$

$-0.6357$

$-0.6439$

$-0.6439$

$-0.6503$

$-0.6679$

$-0.6794$

$-0.6930$

$-0.7007$

$-0.7055$

$-0.7072$

$-1.4813$

$-1.4862$

$-1.5010$

$-1.5264$

$-1.5636$

$-1.0146$

$-1.6820$

$-1.7701$

$-2.0369$

$-2.1311$

$-2.1730$

-2.1950
-2.2177 $\frac{\mathrm{z}}{\mathrm{h}}=0.125$

\begin{tabular}{cc}
\multicolumn{2}{c}{$\Psi_{\mathrm{dD}}(\mathrm{z})$} \\
5.8248 & -0.1004 \\
5.8149 & -0.1003 \\
5.7847 & -0.1003 \\
5.7324 & -0.1002 \\
5.6547 & -0.1001 \\
5.5457 & -0.0999 \\
5.3953 & -0.0997 \\
5.1847 & -0.0995 \\
4.8741 & -0.0992 \\
4.3544 & -0.0989 \\
3.9089 & -0.0987 \\
3.6579 & -0.0987 \\
3.5106 & -0.0986 \\
3.3491 & -0.0986
\end{tabular}

$\Psi_{\mathrm{dI}}^{\prime}(\mathrm{z})$
-0.7432
-0.7430
-0.7426
-0.7420
-0.7410
-0.7398
-0.7383
-0.7366
-0.7346
-0.7323
-0.7311
-0.7305
-0.7303
-0.7300

$\Psi_{\mathrm{dUI}}^{\prime}(\mathrm{z})$

$-0.3832$

$-0.3831$

-0.3829
-0.3826

$-0.3821$

$-0.3815$

$-0.3807$

$-0.3798$

$-0.3798$

$-0.3788$

$-0.3776$

$-0.3770$

$-0.3767$

$-0.3766$

$-0.3764$

$\frac{\mathrm{z}}{\mathrm{h}}=0.250$

$\begin{array}{ll}6.2120 & -0.6519 \\ 6.2007 & -0.6514 \\ 6.1661 & -0.6499 \\ 6.1066 & -0.6475 \\ 6.0188 & -0.6441 \\ 5.8969 & -0.6398 \\ 5.7308 & -0.6346 \\ 5.5019 & -0.6285 \\ 5.1702 & -0.6215 \\ 4.6268 & -0.6136 \\ 4.1683 & -0.6094 \\ 3.9119 & -0.6076 \\ 3.7617 & -0.6067 \\ 3.5973 & -0.6058\end{array}$

$-2.161$

$-2.1595$

$-2.1547$

$-2.1466$

$-2.1355$

$-2.1211$

$-2.0834$

$-2.0601$

$-2.0340$

$-2.0199$

$-2.0141$

$-2.0111$

$-0.6058$

$-2.0081$

$-2.1611$

$-2.1595$

$-2.1547$

$-2.1466$

$-2.1211$

$-2.1211$

$-2.0834$

$-2.0601$

$-2.0340$

$-2.0199$

$-2.0141$

$-2.0111$

$$
\frac{z}{h}=0.375
$$

6.2425

6.2291

6.1882

6.1182

6.0159

5.8755

5.6872

5.4324

5.0714

.4953

4.0191

3.7552

3.6012
3.4328

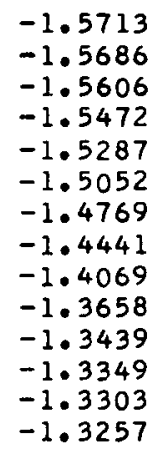

$-2.6750$

$-4.0965$

$-4.0893$

$-4.0679$

$-4.0324$

$-3.9832$

$-3.9206$

$-3.8453$

-3.7578
-3.6589

$-3.5495$

$-3.4911$

$\begin{array}{ll}-2.2862 & -3.4671 \\ -2.2787 & -3.4550 \\ -2.2712 & -3.4428\end{array}$

$\begin{array}{ll}-2.2862 & -3.4671 \\ -2.2787 & -3.4550 \\ -2.2712 & -3.4428\end{array}$ 
$\Omega=8.54$

$\Psi_{d}(z)$

$k_{0} z$

0.01

0.02

0.03

0.04
0.06

0.08

0.10

0.15

0.20

0.30

0.40
0.50

0.60

0.70

0.80

0.90

0.95

0.97

0.98
0.99

0.00

0.10
0.20

0.20
0.25

0.25
0.28

0.28
0.29

0.30

0.31

0.32

0.33

0.34
0.35

0.36

0.37

0.38

0.40

0.45

0.50

0.60
0.70

0.70
0.80

0.90

0.95

0.97

0.98

0.99
$-49.5064$

$-24.7551$

$-16.5055$

$-12.3814$

$-8.2585$

$-6.1984$

$-4.9633$

$-3.3196$

$-2.5011$

$-1.6895$

$-1.2911$

$-1.0587$

$-0.9102$

$-0.8106$

$-0.7429$

$-0.6980$

$-0.6775$

$-0.6752$

$-0.6731$

6.3579
6.4350
6.8070
7.4031
8.3314
8.9335
9.9014
11.7055
16.2277
47.9302
-15.4990
-2.8230
0.3397
1.7726
2.5877
3.4762
4.3354
4.6460
4.8378
4.8114
4.6398
4.2493
3.8699
3.6457
3.5116
3.3631

$-3.4268$

$-3.6924$

$-4.9338$

$-6.8653$

$-9.8285$

$-11.7392$

$-14.8031$

$-20.5020$

$-34.7639$

65.1509

25.1999

15.2179

10.6845

8.0969

5.2560
2.4302

2.4302

1.3108
0.3398

0.3398
-0.1063

$-0.3776$

$-0.5794$

$-0.6698$

$-0.7053$

$-0.7230$

$-0.7408$
$\Psi_{\mathrm{dU}}(\mathrm{z})$

$6.1096-2.2230$

6.1096

6.1096

6.1096

. 1096

6.1097

6.1098

6.1102

6.1110

6.1265

6.1542

6.2188

6.3739

6.7965
8.4536

12.2976

17.7054

24.5908

45.4451

$\Omega=8.54$

6.3579

6.4350

$6.807 \mathrm{C}$

. 4031

8.3314

8.9335

9.9014

11.7055

47.9302

$-15.4990$

-15.4990
-2.8230

0.3397

1.7726
2.5877

3.4762

4.3354

4.6460

4.8378

4.8114

4.6398

4. 2493

3.8699

3.5116

3.3631
-2.2230
-2.2236

$-2.2246$

$-2.2260$

$-2.2300$

$-2.2356$

$-2.2429$

$-2.2685$

$-2.3056$

$-2.4194$

$-2.6013$

$-2.8849$

$-3.3396$

$-4.1293$

$-5.7484$

$-10.6713$

$-20.5597$

33.7563

$-50.2556$

$-99.7584$

-3.4268
-3.6924
-4.9338
-6.8653
-0.8285
-11.7392
-14.8031
-20.5020
-34.7639
-134.6527
65.1509
25.1999
15.2179
10.6845
8.0969
5.2560
2.4302
1.3108
0.3398
-0.1063
-0.3776
-0.5794
-0.6698
-0.7053
-0.7230
-0.7408

$\frac{\mathrm{z}}{\mathrm{h}}=0.500$

$\dot{\Psi}_{\mathrm{dD}}^{(z)}$

$\Psi_{\mathrm{dI}}^{\mathrm{t}}(\mathrm{z}) \quad \dot{\Psi}_{\mathrm{dUI}}^{\prime}(\mathrm{z})$

$5.7793 \quad-2.3957$

$5.7788 \quad-2.3955$

$5.7781-2.3952$

$5.7772 \quad-2.3947$

$5.7743 \quad-2.3932$

$5.7704-2.3912$

$5.7653 \quad-2.3886$

$5.7475-2.3797$

$\begin{array}{ll}5.7225 & -2.3673 \\ 5.6492 & -2.3320\end{array}$

$5.5423-2.2834$

$5.3963-2.2220$

$5.2011-2.1489$

$4.9385-2.0649$

$3.9826 \quad-1.8696$

$3.5007-1.8161$

$3.2341-1.7943$

$3.0786-1.7833$

2.9084

$-1.7722$

$-1.5552$

$-1.5552$

$-1.5550$

$-1.5549$

$-1.5544$

$-1.5537$

-1.5529
-1.5499

$-1.5457$

$-1.5340$

$-1.5177$

$-1.4972$

$-1.4727$

$-1.4446$

$-1.4131$

$-1.3789$

$-1.3608$

$-1.3535$

$-1 \cdot 3498$

$-1.3460$

$-4.4454$

$-4 \cdot 4449$

$-4.4442$

$-4.4431$

$-4.4401$

-4.4359
-4.4305

$-4.4305$

$-4.3855$

$-4.3113$

$-4.2089$

$-4.0798$

$-3.7494$

$-3.5527$

$-3.3387$

$-3.1803$

$-3.1571$

$-3.1339$

$\frac{z}{h}=0.750$

$\begin{array}{ll}4.5740 & -2.9901 \\ 4.5676 & -2.9771 \\ 4.5476 & -2.9385 \\ 4.5319 & -2.9099 \\ 4.5204 & -2.8899 \\ 4.5162 & -2.8828 \\ 4.5117 & -2.8754 \\ 4.5071 & -2.8678 \\ 4.5023 & -2.8600 \\ 4.4973 & -2.8520 \\ 4.4921 & -2.8437 \\ 4.4866 & -2.8352 \\ 4.4809 & -2.8265 \\ 4.4750 & -2.8176 \\ 4.4688 & -2.8085 \\ 4.4557 & -2.7897 \\ 4.4181 & -2.7391 \\ 4.3726 & -2.6839 \\ 4.2508 & -2.5614 \\ 4.0703 & -2.4259 \\ 3.7853 & -2.2822 \\ 3.2964 & -2.1357 \\ 2.8602 & -2.0634 \\ 2.61 C 4 & -2.0350 \\ 2.4625 & -2.0209 \\ 2.2950 & -2.0069\end{array}$

$-2.0074$

$-1.9589$

$-1.8146$

$-1.7076$

$-1.6325$

$-1.6057$

$-1.5780$

$-1.5494$

$-1.4897$

$\begin{array}{ll}1.4586 & -1.4586 \\ 1.4266 & -1.4266\end{array}$

$1.3938-1.3938$

$1.3602-1.3602$

$1.3257-1.3257$

$1.2543-1.2543$

$0.8505-0.8505$

$0.8505-0.8505$

$-0.1671$

$-0.1671$

$-0.3742$

0.1671

0.7674

1.4250

$-1.7765$

1.7765

1.9219

$-1.9957$

$-2.0702$

2.0702 
$\Omega=8.54$

\begin{tabular}{|c|c|c|}
\hline$k_{o} z$ & \multicolumn{2}{|c|}{$\Psi_{d}(z)$} \\
\hline 0.00 & 6.0391 & -0.8754 \\
\hline 0.05 & $7 \cdot 2097$ & -1.1011 \\
\hline 0.10 & 8.8414 & -1.5426 \\
\hline 0.12 & 9.9392 & -1.8703 \\
\hline 0.14 & 11.7044 & -2.4137 \\
\hline 0.16 & 15.1578 & -3.4966 \\
\hline 0.17 & 18.5792 & -4.5776 \\
\hline 0.18 & 25.3940 & -6.7374 \\
\hline 0.19 & 45.7882 & $-13 \cdot 2131$ \\
\hline 0.21 & -35.6565 & 12.6774 \\
\hline 0.22 & $-15 \cdot 2666$ & 6.2017 \\
\hline $\begin{array}{l}0.23 \\
0.24\end{array}$ & $\begin{array}{l}-8.4588 \\
-5.0473\end{array}$ & $\begin{array}{l}4.0418 \\
2.9608\end{array}$ \\
\hline 0.26 & -1.6229 & 1.8777 \\
\hline 0.28 & 0.1002 & 1.3341 \\
\hline $\begin{array}{l}0.30 \\
0.35\end{array}$ & $\begin{array}{l}1.1407 \\
2.5406\end{array}$ & $\begin{array}{l}1.0061 \\
0.5637\end{array}$ \\
\hline 0.40 & 3.2452 & 0.3365 \\
\hline 0.45 & 3.6639 & 0.1952 \\
\hline 0.50 & 3.9336 & 0.0963 \\
\hline 0.52 & 4.0141 & 0.0642 \\
\hline 0.54 & 4.0824 & 0.0350 \\
\hline 0.56 & 4.1402 & 0.0084 \\
\hline 0.57 & 4.1656 & -0.0042 \\
\hline 0.58 & 4.1888 & -0.0162 \\
\hline 0.59 & 4.2100 & -0.0278 \\
\hline 0.61 & 4.2464 & -0.0499 \\
\hline & 4.2619 & -0.0604 \\
\hline 0.63 & $\begin{array}{l}4.2756 \\
4.2876\end{array}$ & -0.0706 \\
\hline 0.64 & 4.2876 & -0.0805 \\
\hline 0.66 & $4 \cdot 3067$ & -0.0996 \\
\hline 0.68 & 4.3193 & -0.1175 \\
\hline 0.70 & $4 \cdot 3257$ & -0.1354 \\
\hline 0.75 & $4 \cdot 3138$ & -0.1768 \\
\hline 0.80 & $4 \cdot 2580$ & -0.2158 \\
\hline 0.90 & $\begin{array}{l}3.9517 \\
3.6077\end{array}$ & $\begin{array}{l}-0.2917 \\
-0.3304\end{array}$ \\
\hline 0.97 & $\begin{array}{l}3.6011 \\
3.3965\end{array}$ & $\begin{array}{l}-0.3304 \\
-0.3463\end{array}$ \\
\hline 0.98 & $\begin{array}{l}3.3963 \\
3.2688\end{array}$ & -0.3544 \\
\hline 0.99 & 3.1264 & -0.3626 \\
\hline
\end{tabular}

$\Psi_{\mathrm{dU}}(\mathrm{z})$

$\begin{array}{rr}5.9905 & -2.7579 \\ 5.9930 & -2.7744 \\ 6.0009 & -2.8256 \\ 6.0057 & -2.8566 \\ 6.0117 & -2.8943 \\ 6.0190 & -2.9393 \\ 6.0232 & -2.9647 \\ 6.0278 & -2.9922 \\ 6.0328 & -3.0219 \\ 6.0442 & -3.0886 \\ 6.0507 & -3.1258 \\ 6.0578 & -3.1658 \\ 6.0655 & -3.2089 \\ 6.0831 & -3.3052 \\ 6.1041 & -3.4169 \\ 6.1293 & -3.5467 \\ 6.2172 & -3.9775 \\ 6.3644 & -4.6479 \\ 6.6340 & -5.7924 \\ 7.2169 & -8.1157 \\ 7.6720 & -9.8687 \\ 8.4458 & -12.7976 \\ 10.0186 & -18.6656 \\ 11.6049 & -24.5383 \\ 14.7917 & -36.2884 \\ 24.3821 & -71.5474 \\ 14.0748 & 69.5140 \\ -4.4859 & 34.2546 \\ -1.3017 & 22.5041 \\ 0.2810 & 16.6306 \\ 1.8434 & 10.7607 \\ 2.6025 & 7.8290 \\ 3.0383 & 6.0724 \\ 3.5509 & 3.7364 \\ 3.7123 & 2.5739 \\ 3.5468 & 1.4188 \\ 3.2163 & 1.0904 \\ 3.0072 & 0.9839 \\ 2.3808 & 0.9349 \\ 2.7400 & 0.8884\end{array}$

$$
\frac{\mathrm{z}}{\mathrm{h}}=0.625
$$

$\begin{array}{ll} & \Psi \mathrm{dD}^{(z)} \\ 5.1207 & -2.8379 \\ 5.1179 & -2.8350 \\ 5.1094 & -2.8262 \\ 5.1044 & -2.8211 \\ 5.0984 & -2.8150 \\ 5.0915 & -2.8080 \\ 5.0877 & -2.8042 \\ 5.0836 & -2.8001 \\ 5.0793 & -2.7958 \\ 5.0700 & -2.7866 \\ 5.0649 & -2.7816 \\ 5.0596 & -2.7764 \\ 5.0540 & -2.7710 \\ 5.0421 & -2.7596 \\ 5.0291 & -2.7472 \\ 5.0150 & -2.7340 \\ 4.9747 & -2.6973 \\ 4.9266 & -2.6554 \\ 4.8698 & -2.6086 \\ 4.8034 & -2.5572 \\ 4.7738 & -2.5353 \\ 4.7423 & -2.5128 \\ 4.7088 & -2.4896 \\ 4.6912 & -2.4778 \\ 4.6731 & -2.4658 \\ 4.6544 & -2.4536 \\ 4.6152 & -2.4288 \\ 4.5946 & -2.4162 \\ 4.5733 & -2.4035 \\ 4.5514 & -2.3905 \\ 4.5052 & -2.3643 \\ 4.4557 & -2.3375 \\ 4.4026 & -2.3102 \\ 4.2512 & -2.2397 \\ 4.0654 & -2.1665 \\ 3.5133 & -2.0132 \\ 3.0478 & -1.9339 \\ 2.7873 & -1.9019 \\ 2.6345 & -1.8858 \\ 2.4668 & -1.8697\end{array}$

$\begin{array}{cc}\Psi_{\mathrm{dI}}^{1}(\mathrm{z}) & \Psi_{\mathrm{dUI}}^{\prime}(\mathrm{z}) \\ 0.4477 & -3.4049 \\ 0.4440 & -3.3987 \\ 0.4330 & -3.3800 \\ 0.4265 & -3.3690 \\ 0.4189 & -3.3561 \\ 0.4101 & -3.3412 \\ 0.4053 & -3.3330 \\ 0.4002 & -3.3244 \\ 0.3948 & -3.3152 \\ 0.3832 & -3.2955 \\ 0.3770 & -3.2849 \\ 0.3704 & -3.2739 \\ 0.3636 & -3.2624 \\ 0.3492 & -3.2379 \\ 0.3337 & -3.2116 \\ 0.3171 & -3.1834 \\ 0.2710 & -3.1051 \\ 0.2184 & -3.0158 \\ 0.1596 & -2.9160 \\ 0.0949 & -2.8062 \\ 0.0674 & -2.7596 \\ 0.0391 & -2.7115 \\ 0.0099 & -2.6620 \\ -0.0050 & -2.6367 \\ -0.0201 & -2.6110 \\ -0.0355 & -2.5851 \\ -0.0667 & -2.5321 \\ -0.0826 & -2.5051 \\ -0.0987 & -2.4778 \\ -0.1150 & -2.4502 \\ -0.1481 & -2.3941 \\ -0.1820 & -2.3368 \\ -0.2165 & -2.2783 \\ -0.3057 & -2.1273 \\ -0.3988 & -1.9700 \\ -0.5951 & -1.6394 \\ -0.6976 & -1.4675 \\ -0.7394 & -1.3978 \\ -0.7604 & -1.3627 \\ -0.7815 & -1.3275\end{array}$




\begin{tabular}{|c|c|c|}
\hline$k_{0} z$ & \multicolumn{2}{|c|}{$\Psi_{d}(z)$} \\
\hline 0.00 & 7.0953 & -6.7603 \\
\hline 0.10 & 5.3242 & -4.6700 \\
\hline 0.15 & 5.0194 & -4.2555 \\
\hline 0.20 & 4.8069 & -4.0659 \\
\hline 0.22 & 4.7318 & $-4 \cdot 0482$ \\
\hline 0.24 & 4.6574 & -4.0671 \\
\hline 0.25 & 4.6194 & -4.0922 \\
\hline 0.26 & 4.5801 & $-4 \cdot 1291$ \\
\hline 0.27 & 4.5391 & $-4 \cdot 1794$ \\
\hline 0.28 & 4.4956 & -4.2452 \\
\hline 0.29 & 4.4489 & -4.3291 \\
\hline 0.30 & 4.3978 & -4.4347 \\
\hline 0.31 & 4.3411 & -4.5670 \\
\hline 0.32 & 4.2769 & $-4 \cdot 7326$ \\
\hline 0.34 & 4.1152 & -5.2061 \\
\hline 0.36 & 3.8748 & -6.0025 \\
\hline 0.38 & 3.4563 & -7.5127 \\
\hline 0.39 & 3.0932 & -8.8798 \\
\hline 0.40. & 2.4836 & -11.2243 \\
\hline $0.41^{\circ}$ & 1.2290 & $-16 \cdot 1232$ \\
\hline 0.42 & -2.9283 & $-32 \cdot 5133$ \\
\hline 0.43 & 50.9703 & $18 \mathrm{C} \cdot 9008$ \\
\hline 0.44 & 10.5281 & 20.8805 \\
\hline 0.45 & 7.8222 & 10.2328 \\
\hline 0.46 & 6.8316 & 6.3739 \\
\hline 0.48 & 5.9936 & 3.1803 \\
\hline 0.50 & 5.6125 & 1.7944 \\
\hline 0.55 & 5.1745 & $0.368 E$ \\
\hline 0.60 & 4.9515 & $-0.192 \epsilon$ \\
\hline 0.70 & 4.6460 & -0.655 \\
\hline & 4.3338 & 854 \\
\hline 0.90 & 3.8584 & -0.9785 \\
\hline & 3.4477 & -1.0331 \\
\hline 0.97 & 3.2124 & -1.0550 \\
\hline $\begin{array}{l}0.98 \\
0.99\end{array}$ & $\begin{array}{l}3.0731 \\
2.9194\end{array}$ & $\begin{array}{l}-1.0662 \\
-1.0774\end{array}$ \\
\hline
\end{tabular}

$\Omega=8.54$

$$
\frac{\mathrm{z}}{\mathrm{h}}=0.87 \mathrm{j}
$$

$$
\Psi_{\mathrm{dD}}(z)
$$

8.4323
11.2436

$-4.5854$ $-7.5854$ $-23.1033 \quad 31.4096$ $2.4293 \quad 2.2256$ $3.3413 \quad 1.1740$ $3.8654 \quad 0.5645$ $4.0501 \quad 0.3476$ $4.2011 \quad 0.1689$ $4.3265 \quad 0.0194$ $4.4317-0.1076$ $4.5211 \quad-0.2167$ $4.5977 \quad-0.3114$ $4.6639-0.3945$ $4.7213-0.4681$ $4.8155 \quad-0.5924$ $4.8887 \quad-0.6939$ $4.9460 \quad-0.7789$ $4.9700 \quad-0.8165$ $4.9914 \quad-0.8515$ $5.0104-0.8841$ $5.0272 \quad-0.9148$ $5.0422-0.9436$ $5.0555-0.9709$ $5.0672-0.9969$ $5.0775-1.0217$ $5.0943-1.0682$ $5.1066-1.1116$ $5.1213,-1.2110$ $5.1172-1.3042$ $5.0601-1.4969$ $4.9267-1.7337$ $4.6414 \quad-2.0722$ $4.1643 \quad-2.4292$ $\begin{array}{ll}4.0513 & -2.4932 \\ 3.9235 & -2.5615\end{array}$

\begin{tabular}{cc}
$\Psi_{\mathrm{dI}}^{\prime}(\mathrm{z})$ & $\Psi^{\prime}{ }^{\mathrm{d} U I}{ }^{(\mathrm{z})}$ \\
2.4847 & -0.6981 \\
2.4073 & -0.6024 \\
2.3109 & -0.4832 \\
2.1767 & -0.3171 \\
2.1125 & -0.2377 \\
2.0424 & -0.1510 \\
2.0051 & -0.1049 \\
1.9664 & -0.0570 \\
1.9263 & -0.0073 \\
1.8846 & 0.0443 \\
1.8416 & 0.0976 \\
1.7971 & 0.1527 \\
1.7511 & 0.2095 \\
1.7038 & 0.2682 \\
1.6048 & 0.3907 \\
1.5002 & 0.5203 \\
1.3900 & 0.6568 \\
1.3329 & 0.7276 \\
1.2743 & 0.8002 \\
1.2144 & 0.8745 \\
1.1531 & 0.9505 \\
1.0904 & 1.0282 \\
1.0264 & 1.1077 \\
0.9610 & 1.1888 \\
0.8943 & 1.2717 \\
0.7567 & 1.4425 \\
0.6137 & 1.6203 \\
0.2322 & 2.0950 \\
-0.1843 & 2.6145 \\
-1.1307 & 3.8022 \\
-2.2645 & 5.2414 \\
-3.6896 & 7.0835 \\
-4.5861 & 8.2626 \\
-4.9973 & 8.8087 \\
-5.2173 & 9.1019 \\
-5.4481 & 9.4105 \\
& \\
\hline &
\end{tabular}




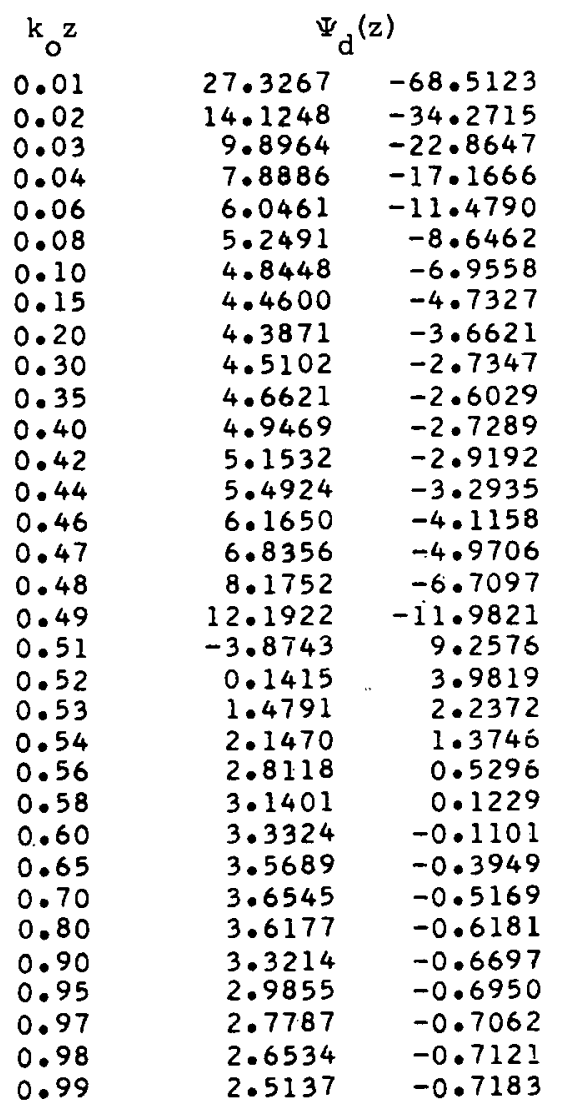

$\Omega=8.54$

$$
\frac{\mathrm{z}}{\mathrm{h}}=1.000
$$

$$
\Psi_{\mathrm{dU}}{ }^{(\mathrm{z})}
$$

$-283.3102-309.2003$

$-66.8294$

$-26.7405$

$-12.7096$

$-2.6880$

-78.4937
-35.7708

$-20.8185$

$-10.1401$

$-6.4053$

-4.6796
-2.9867

-2.986 .7
-2.4118

-2.4118
-2.0556

$-2.0153$

$-2.0194$

$-2.0308$

$-2.0472$

$-2.0686$

$-2.0812$

$-2.0949$

$-2.1100$

$-2.1439$

$-2.1628$

$-2.1831$

$-2.2049$

$-2.2530$

$-2.3078$

$-2.3698$

$-2.5639$

-2.8333
-3.8108

$-6.8058$

$-12.8268$

$-20.8617$

$-30.9074$

$-61.0466$

$$
\Psi \mathrm{dD}(\mathrm{z})
$$

3.9048

3.9047
3.9045

3.9045
3.9043

3.9036

3.9026

3.9013

3.8965

3.8893

3.8653

3.8469

3.8229

3.8114

3. 7987

3.7847

3.7692

3. 7609

3.7431

3.7336
3.7235

3.7130

3.6905

3.6657

3.6386

3.5586

3.4574
3.1599

3.1599

2.6022

1. 5034

1.5034

1.0009
-0.3042
$-3.1498$

$-3.1495$

$-3.1491$

$-3.1484$

$-3.1467$

$-3.1442$

$-3.1305$

$-3.1163$

$-3.0798$

$-3.0595$

-3.0400
-3.0327

$-3.0261$

$-3.0203$

$-3.0177$

$-3.0134$

$-3.0105$

$-3.0096$

$-3.0091$

$-3.0110$

$-3.0154$

$-3.0229$

$-3.0597$

-3.1341
-3.5195

$-5.0310$

$-8.3291$

$-12.8122$

$-18.4434$

$-35.3735$

$\begin{array}{rr}\Psi_{\mathrm{dI}}^{\prime}(\mathrm{z}) & \Psi_{\mathrm{dUI}}^{\prime}(\mathrm{z}) \\ 2.1515 & 0.3051 \\ 2.1498 & 0.3098 \\ 2.1470 & 0.3175 \\ 2.1430 & 0.3283 \\ 2.1317 & 0.3592 \\ 2.1159 & 0.4025 \\ 2.0956 & 0.4581 \\ 2.0247 & 0.6511 \\ 1.9253 & 0.9212 \\ 1.6381 & 1.6947 \\ 1.4483 & 2.2008 \\ 1.2254 & 2.7907 \\ 1.1263 & 3.0515 \\ 1.0211 & 3.3273 \\ 0.9096 & 3.6187 \\ 0.8513 & 3.7706 \\ 0.7913 & 3.9268 \\ 0.7294 & 4.0874 \\ 0.6001 & 4.4224 \\ 0.5325 & 4.5972 \\ 0.4628 & 4.7772 \\ 0.3908 & 4.9625 \\ 0.2399 & 5.3504 \\ 0.0788 & 5.7633 \\ -0.0937 & 6.2043 \\ -0.5851 & 7.4558 \\ -1.1927 & 8.9974 \\ -3.0782 & 13.7876 \\ -8.0431 & 26.5570 \\ -17.4434 & 50.9912 \\ -29.8151 & 83.2617 \\ -45.2285 & 123.5074 \\ -91.4013 & 244.1260\end{array}$


$\Omega=9.92$

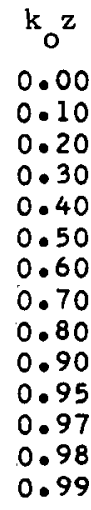

$\Psi_{d}(z)$

6.8522
7.1359

7.2516

7.2989

7.2963

7.2470

7.1452

5.9730

S.6862

6.1492

5.6246

5.2839

5.0574

4.7761

0.99

0.00

0.10

0.20

0.30

0.40

0.50

0.60

0.70

0.80

0.90

0.95

0.97

0.98

0.99

4.7696

$\begin{array}{ll}7.5846 & -0.6331 \\ 7.5761 & -0.6337 \\ 7.5500 & -0.6358 \\ 7.5044 & -0.6392 \\ 7.4358 & -0.6440 \\ 7.3380 & -0.6504 \\ 7.2003 & -0.6583 \\ 7.0017 & -0.6680 \\ 6.6965 & -0.679 .5 \\ 6.1479 & -0.6931 \\ 5.6198 & -0.7008 \\ 5.2781 & -0.7040 \\ 5.0512 & -0.7057 \\ 4.7696 & -0.7073\end{array}$

$-0.7073$

$\begin{array}{lll}0.00 & 8.6839 & -2.3362 \\ 0.10 & 7.6674 & -1.9125 \\ 0.20 & 7.2875 & -1.6483 \\ 0.30 & 7.0728 & -1.4691 \\ 0.40 & 6.9147 & -1.3412 \\ 0.50 & 6.7677 & -1.2472 \\ 0.60 & 6.6032 & -1.1770 \\ 0.70 & 6.3915 & -1.1247 \\ 0.80 & 6.0823 & -1.0868 \\ 0.90 & 5.5361 & -1.0670 \\ 0.95 & 5.0113 & -1.0523 \\ 0.97 & 4.6713 & -1.0495 \\ 0.98 & 4.4453 & -1.0482 \\ 0.99 & 4.1646 & -1.0471\end{array}$

\subsection{0}

7.6856

7.6691

7.6402
7.5961

7.5961
7.5322

7.5322
7.4398

7.3012

7.0753

6.6324

6.1697

5.8563

5.6435

$\Psi_{\mathrm{dU}}(\mathrm{z})$

$-0.0996$

$-0.0996$

$-0.0996$

$-0.0997$

$-0.0999$

-0.1001
-0.1004

$-0.1007$

$-0.1010$

$-0.1014$

$-0.1016$

$-0.1017$

$-0.1017$

$-0.1018$

$-0.6331$

$-0.6358$

$-0.6392$

$-0.6440$

$-0.6504$

$-0.6583$

$-0.6680$

$-0.6795$

$-0.6931$

$-0.7008$

$-0.7040$

$-0.7057$

$-0.7073$

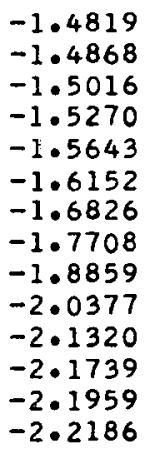

$\frac{\mathrm{z}}{\mathrm{h}}=0.125$

$\Psi_{\mathrm{dD}}{ }^{(\mathrm{z})}$

7.1770 $\mathrm{dD}^{(\mathrm{z})}$

$7.1668 \quad-0.1003$

$7.1357 \quad-0.1003$

$7.0015 \quad-0.1001$

$6.8886 \quad-0.0999$

$6.7320 \quad-0.0997$

$6.5108 \quad-0.0995$

$6.1785 \quad-0.0992$

$5.5997-0.0989$

$\begin{array}{ll}5.0552 & -0.0987\end{array}$

$4.7069-0.0987$

$4.4770 \quad-0.0986$

4.1927

$-0.0986$

$\begin{array}{cc}\Psi_{\mathrm{dI}}^{\prime}(\mathrm{z}) & \Psi_{\mathrm{dUI}}^{\prime}(\mathrm{z}) \\ -0.7432 & -0.3832 \\ -0.7431 & -0.3831 \\ -0.7427 & -0.3829 \\ -0.7420 & -0.3826 \\ -0.7410 & -0.3821 \\ -0.7398 & -0.3815 \\ -0.7383 & -0.3807 \\ -0.7366 & -0.3798 \\ -0.7346 & -0.3788 \\ -0.7323 & -0.3776 \\ -0.7311 & -0.3770 \\ -0.7306 & -0.3767 \\ -0.7303 & -0.3766 \\ -0.7301 & -0.3764\end{array}$

$$
\frac{z}{h}=0.250
$$

$\begin{array}{ll}7.5668 & -0.6520 \\ 7.5551 & -0.6515 \\ 7.5197 & -0.6500 \\ 7.4586 & -0.6476 \\ 7.3682 & -0.6442 \\ 7.2424 & -0.6399 \\ 7.0701 & -0.6347 \\ 6.8305 & -0.6286 \\ 6.4777 & -0.6216 \\ 5.8747 & -0.6137 \\ 5.3173 & -0.6095 \\ 4.9637 & -0.6077 \\ 4.7310 & -0.6068 \\ 4.4439 & -0.6059\end{array}$

$-2.1615$

$-2.1598$

$-2.1550$

$-2.1470$

$-2.1358$

$-2.1215$

$-2.0837$

$-2.0604$

$-2.0343$

$-2.0202$

$-2.0144$

$-2.0114$

$-2.0085$

$-2.1615$

$-2.1598$

-2.1550
-2.1470

$-2 \cdot 1358$

$-2.1215$

$-2.1041$

$-2.0837$

$-2.0604$

$-2.0343$

$-2.0202$

$-2.0144$

$-2.0114$

$$
\frac{z}{h}=0.375
$$

$\begin{array}{ll}7.6018 & -1.5719 \\ 7.5880 & -1.5692 \\ 7.5463 & -1.5612 \\ 7.4747 & -1.5478 \\ 7.3698 & -1.5293 \\ 7.2255 & -1.5058 \\ 7.0310 & -1.4775 \\ 6.7656 & -1.4446 \\ 6.3834 & -1.4075 \\ 5.7480 & -1.3664 \\ 5.1732 & -1.3445 \\ 4.8123 & -1.3354 \\ 4.5758 & -1.3309 \\ 4.2848 & -1.3263\end{array}$

-2.6760
-2.6716
-2.6584
-2.6365
-2.6061
-2.5674
-2.5209
-2.4668
-2.4057
-2.3381
-2.3020
-2.2872
-2.2797
-2.2722

-4.0980
-4.0909
-4.0695
-4.0340
-3.9847
-3.9222
-3.8462
-3.7593
-3.6604
-3.5510
-3.4926
-3.4686
-3.4565
-3.4443


$\Omega=9.92$

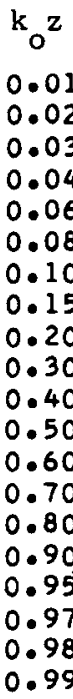

$\mathrm{k}_{\mathrm{o}} \mathrm{z}$

0.01
0.02

0.03

0.04

0.10

0.15

0.20

0.30

0.50

0.70

0.80

.90

0.97

0.98

0.99

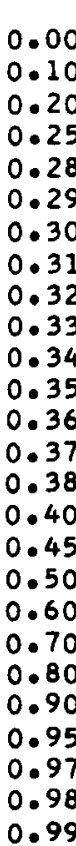

$\Psi_{\mathrm{d}}(\mathrm{z})$

23.0072

12.3843

9.1017

7.5994

6.2934

5.7789

5.5488

5.3964
5.4266

5.4266
5.5680

5.6815

5.7394

5.7345

5.6523

5.4523

5.0027

4.523

4.2010

3.9839

3.7120

$-49.5524$

$-24.7781$

$-16.5209$

$-8.2662$

$-6.2041$

$-4.9679$

$-3.3227$

$-2.5034$

$-1.6910$

$-1.2923$

$-1.0597$

$-0.9111$

$-0.8114$

$-0.7437$

$-0.6987$

$-0.6832$

$-0.6782$

$-0.6759$

$-0.6738$

$4.4887 \quad-0.7233$

$-0.7411$
$\Psi_{\mathrm{dU}}{ }^{(\mathrm{z})}$

7.4921

7.4921

7.4921

7.4921

7.4922

7.4922

7.4923
7.4928

7.4938

7.4983

7.5104

7.5394

7.6063

7.7661

8.2010

9.9122

13.9161

19.5972

26.8733

49.0196

$\Omega=9.92$

7.8031

7.8883

8.2984

9.9753

.6372

1.7012

13.6842

18.6542

53.4951

$-16.2124$

$-2.2813$

1.1948

2.7699

3.6660

4.6431

5.5893

5.9328

6.1488

6.1257

5.9413

5.4983

5.0238

4.7043

4.4887

4.2186 $\frac{z}{h}=0.500$

\begin{tabular}{cc}
\multicolumn{2}{c}{$\Psi \mathrm{dD}^{(z)}$} \\
7.1447 & -2.3976 \\
7.1443 & -2.3974 \\
7.1436 & -2.3970 \\
7.1426 & -2.3965 \\
7.1397 & -2.3951 \\
7.1357 & -2.3931 \\
7.1305 & -2.3905 \\
7.1124 & -2.3816 \\
7.0868 & -2.3692 \\
7.0120 & -2.3339 \\
6.9026 & -2.2852 \\
6.7526 & -2.2238 \\
6.5514 & -2.1507 \\
6.2782 & -2.0667 \\
5.8874 & -1.9731 \\
5.2424 & -1.8714 \\
4.6623 & -1.8178 \\
4.2991 & -1.7960 \\
4.0613 & -1.7850 \\
3.7687 & -1.7739
\end{tabular}

$\Psi_{\mathrm{dI}}^{\prime}(z)$

$\Psi_{\mathrm{dUI}}^{\prime}(\mathrm{z})$

$-1.5567$

$-1.5566$

$-1.5565$

$-1.5563$

$-1.5558$

$-1.5552$

$-1.5513$

-1.5472
-1.5354

$-1.5192$

$-1.4987$

$-1.4741$

$-1.4460$

$-1.4145$

$-1.3622$

$-1.3549$

$-1.3512$

$-33.7871$

-50.3018
-99.8505

3.7687

$-1.7739$

$\frac{z}{h}=0.750$

$-3.4314$

$-3.6974$

$-4.9407$

$-6.8752$

$-9.8430$

.7567

$-14.8253$

$-20.5331$

$-134.8610$

65.2527

25.2397

15.2422

10.7018

8.1102

5. 2649

2.4347

1.3135

0.3410

$-0.1057$

$-0.3774$

$-0.5795$

$-0.6700$

$-0.7056$

$-0.7233$

$-0.7411$

$\begin{array}{ll}5.9562 & -2.9970 \\ 5.9496 & -2.9840 \\ 5.9289 & -2.9454 \\ 5.9126 & -2.9168 \\ 5.9007 & -2.8967 \\ 5.8963 & -2.8896 \\ 5.8918 & -2.8822 \\ 5.8870 & -2.8746 \\ 5.8820 & -2.8668 \\ 5.8768 & -2.8588 \\ 5.8714 & -2.8505 \\ 5.8657 & -2.8420 \\ 5.8598 & -2.8333 \\ 5.8537 & -2.8244 \\ 5.8473 & -2.8153 \\ 5.8337 & -2.7965 \\ 5.7947 & -2.7459 \\ 5.7473 & -2.6907 \\ 5.6204 & -2.5680 \\ 5.4305 & -2.4326 \\ 5.1306 & -2.2889 \\ 4.5825 & -2.1424 \\ 4.0519 & -2.0702 \\ 3.7079 & -2.0418 \\ 3.4792 & -2.0277 \\ 3.1948 & -2.0138\end{array}$

$\begin{array}{ll}2.0101 & -2.0101 \\ 1.9616 & -1.9616 \\ 1.8172 & -1.8172 \\ 1.7100 & -1.7100 \\ 1.6349 & -1.6349 \\ 1.6081 & -1.6081 \\ 1.5804 & -1.5804 \\ 1.5518 & -1.5518 \\ 1.5223 & -1.5223 \\ 1.4920 & -1.4920 \\ 1.4609 & -1.4609 \\ 1.4289 & -1.4289 \\ 1.3960 & -1.3960 \\ 1.3624 & -1.3624 \\ 1.3279 & -1.3279 \\ 1.2564 & -1.2564 \\ 1.0639 & -1.0639 \\ 0.8523 & -0.8523 \\ 0.3756 & -0.3756 \\ -0.1661 & 0.1661 \\ -0.7670 & 0.7670 \\ -1.4252 & 1.4252 \\ 1.7771 & 1.7771 \\ -1.9227 & 1.9227 \\ -1.9965 & 1.9965 \\ -2.0711 & 2.0711\end{array}$

$-4.4487$ $-4.4475$ $-4.4464$ $-4.4434$ $-4.4338$ $-4.4150$ $-4 \cdot 3888$ $-4.2122$ $-4.0830$ $-3.9291$ $-3.5557$ $-3.3417$ $-3.2291$ $-3.1832$ -3.1600
-3.1368

3.1948

$-2.0711$

2.0711 
$\Omega=9.92$

$\Psi_{d}(z)$

$k_{0} z$
0.00
0.05
0.10
0.12
0.14
0.16
0.17
0.18
0.19
0.21
0.22
0.23
0.24
0.26
0.28
0.30
0.35
0.40
0.45
0.50
0.52
0.54
0.56
0.57
0.58
0.59
0.61
0.62
0.63
0.64
0.66
0.68
0.70
0.75
0.80
0.90
0.95
0.97
0.98
0.99
$\Psi_{\mathrm{dU}}(\mathrm{z})$

7.3960
7.3988

7.4076

7.4130

7.4197
7.4278

7.4325

7.4376

7.4431

7.4558

7.4630

7.4708

7.4793

7.4988

7.5219

7.5495

7.6458

7.8065

8.0999

8.7325

9.2258

10.0640

11.7669

13.4840

16.9332

27.3123

$-14.3050$

$-3.9275$

$-0.4811$

-0.4811
1.2321

2.9237

3.7459

4. 2182

4.7741

4. 9482

4. 7462

4.3206

4.0143

3.8050
3.5419 $\frac{\mathrm{z}}{\mathrm{h}}=0.650$

$\Psi_{\mathrm{dD}}(\mathrm{z})$

$-2.7611$

-2.7777
-2.8289

$-2.8600$

$-2.8978$

$-2.9428$

$-2.5682$

$-2.5958$

-3.0255
-3.0923

$-3.1295$

$-3.1697$

$-3.2128$

$-3.3092$

$-3.4210$

$-3.5510$

$-3.9825$

$-4.6538$

$-5.7999$

$-8.1264$

$-9.8819$

$-12.8149$

$-18.6911$

$-24.5721$

$-36.3387$

$-71.6472$

69.6122

34.3034

22.5363

16.6546

10.7764

7.8406

6.0816

3.7423

2.5782

1.4215

1.0926

0.9860

0.9369

0.8904

$\begin{array}{ll}6.4939 & -2.8419 \\ 6.4910 & -2.8390 \\ 6.4823 & -2.8302 \\ 6.4771 & -2.8251 \\ 6.4710 & -2.8190 \\ 6.4640 & -2.8120 \\ 6.4601 & -2.8082 \\ 6.4559 & -2.8041 \\ 6.4515 & -2.7958 \\ 6.4419 & -2.7906 \\ 6.4367 & -2.7856 \\ 6.4313 & -2.7804 \\ 6.4256 & -2.7750 \\ 6.4134 & -2.7635 \\ 6.4000 & -2.7512 \\ 6.3856 & -2.7380 \\ 6.3442 & -2.7012 \\ 6.2948 & -2.6593 \\ 6.2364 & -2.6125 \\ 6.1679 & -2.5610 \\ 6.1373 & -2.5392 \\ 6.1048 & -2.5167 \\ 6.0701 & -2.4935 \\ 6.0519 & -2.4816 \\ 6.0331 & -2.4696 \\ 6.0137 & -2.4574 \\ 5.9730 & -2.4327 \\ 5.9516 & -2.4200 \\ 5.9295 & -2.4073 \\ 5.9066 & -2.3943 \\ 5.8584 & -2.3681 \\ 5.8066 & -2.3413 \\ 5.7510 & -2.3139 \\ 5.5914 & -2.2435 \\ 5.3933 & -2.1702 \\ 4.7833 & -2.0168 \\ 4.2207 & -1.9376 \\ 3.8643 & -1.9055 \\ 3.6297 & -1.8894 \\ 3.3399 & -1.8733\end{array}$

$\Psi_{\mathrm{dI}}^{\prime}(\mathrm{z})$

$\Psi^{\prime} \mathrm{dUI}(\mathrm{z})$

0.4474

0.4437

0.4327

0.4262

0.4186

0.4050

0.3999

0.3945

0.3829

0.3766

0.3701

0.3633

0.3489

0.3334

0.3168

0.2706

0.2180

0.1591

0.0944

0.0669

0.0386

0.0094

$-0.0056$

$-0.0207$

$-0.0360$

$-0.0673$

$-0.0832$

$-0.0993$

$-0.1156$

$-0.1487$

$-0.1826$

$-0.2171$

$-0.3064$

$-0.3996$

$-0.5960$

$-0.6986$

$-0.7404$

$-0.7404$

-0.7614
-0.7825
$-3.4089$

$-3.4027$

$-3.3840$

$-3.3730$

$-3.3601$

$-3.3452$

$-3.3370$

$-3.3192$

$-3.2995$

$-3.2889$

$-3.2778$

$-3.2663$

$-3.2418$

$-3.2155$

$-3.1873$

$-3.1089$

$-3.0196$

-2.9198
-2.8099

$-2.7633$

$-2.7152$

$-2.6656$

$-2.6403$

$-2.6147$

$-2.5887$

$-2.5357$

$-2.5087$

$-2.4814$

$-2.4538$

$-2.3976$

$-2.3402$

$-2.1306$

$-1.9733$

$-1.6426$

$-1.4706$

$-1.4008$

-1.3657
-1.3305 
$\Omega=9.92$

\begin{tabular}{lrr}
$\mathrm{k}_{\mathrm{o}} \mathrm{z}$ & \multicolumn{2}{c}{$\Psi_{\mathrm{d}}(\mathrm{z})$} \\
0.00 & 8.7336 & -6.7775 \\
0.10 & 6.7908 & -4.6821 \\
0.15 & 6.4796 & -4.2668 \\
0.20 & 6.2688 & -4.0771 \\
0.22 & 6.1966 & -4.0595 \\
0.24 & 6.1266 & -4.0787 \\
0.25 & 6.0915 & -4.1040 \\
0.26 & 6.0557 & -4.1411 \\
0.27 & 6.0187 & -4.1917 \\
0.28 & 5.9800 & -4.2579 \\
0.29 & 5.9389 & -4.3422 \\
0.30 & 5.8945 & -4.4484 \\
0.31 & 5.8459 & -4.5813 \\
0.32 & 5.7914 & -4.7477 \\
0.34 & 5.6564 & -5.2233 \\
0.36 & 5.4594 & -6.0232 \\
0.38 & 5.1215 & -7.5400 \\
0.39 & 4.8306 & -8.9129 \\
0.40 & 4.3443 & -11.2675 \\
0.41 & 3.3467 & -16.1873 \\
0.42 & 0.0477 & -32.6474 \\
0.43 & 42.7788 & 181.6773 \\
0.44 & 10.7109 & 20.9741 \\
0.45 & 8.5626 & 10.2809 \\
0.46 & 7.7743 & 6.4055 \\
0.48 & 7.1040 & 3.1982 \\
0.50 & 6.7959 & 1.8063 \\
0.55 & 6.4329 & 0.3743 \\
0.60 & 6.2384 & -0.1893 \\
0.70 & 5.9507 & -0.6543 \\
0.80 & 5.5309 & -0.8541 \\
0.90 & 5.1055 & -0.9789 \\
0.95 & 4.5008 & -1.0338 \\
0.97 & 4.2706 & -1.0558 \\
0.98 & 4.0500 & -1.0670 \\
0.99 & 3.7749 & -1.0783 \\
& &
\end{tabular}

$\frac{\mathrm{z}}{\mathrm{h}}=0.875$

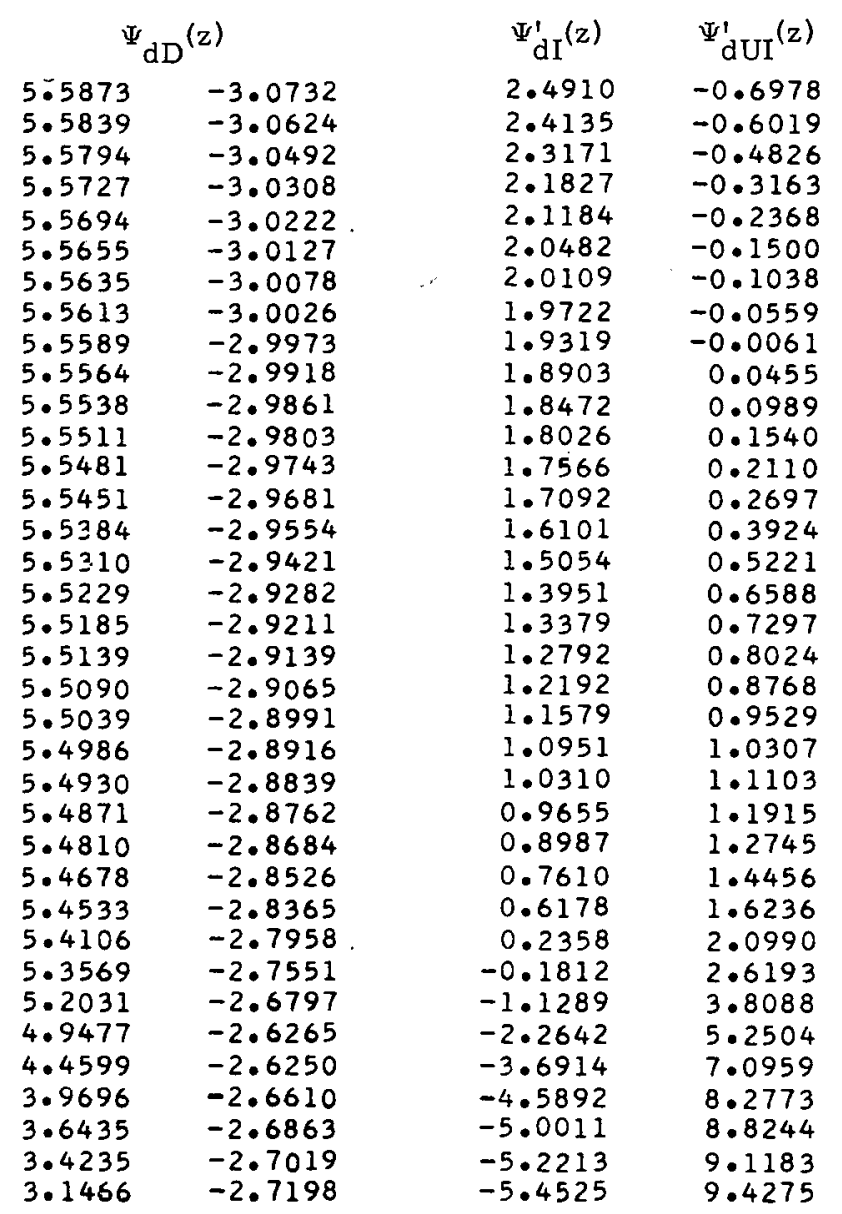

\begin{tabular}{ll}
\multicolumn{2}{c}{$\Psi_{\mathrm{dU}}(\mathrm{z})$} \\
9.9669 & -4.5833 \\
12.9350 & -7.8021 \\
-23.3265 & 31.3722 \\
3.6297 & 2.2201 \\
4.5927 & 1.1696 \\
5.1461 & 0.5608 \\
5.3412 & 0.3441 \\
5.5007 & 0.1657 \\
5.6331 & 0.0163 \\
5.7443 & -0.1106 \\
5.8388 & -0.2196 \\
5.9197 & -0.3142 \\
5.9896 & -0.3973 \\
6.0503 & -0.4707 \\
6.1500 & -0.5950 \\
6.2274 & -0.6964 \\
6.2881 & -0.7813 \\
6.3135 & -0.8189 \\
6.3362 & -0.8538 \\
6.3563 & -0.8865 \\
6.3742 & -0.9171 \\
6.3901 & -0.9459 \\
6.4042 & -0.9732 \\
6.4167 & -0.9992 \\
6.4276 & -1.0240 \\
6.4456 & -1.0705 \\
6.4587 & -1.1139 \\
6.4747 & -1.2133 \\
6.4707 & -1.3066 \\
6.4100 & -1.4995 \\
6.2643 & -1.7366 \\
5.9347 & -2.0758 \\
5.5570 & -2.3165 \\
5.2857 & -2.4335 \\
5.0959 & -2.4977 \\
4.8515 & -2.5662
\end{tabular}

. 
$\Omega=9.92$

$k_{0} z$
0.01
0.02
0.03
0.04
0.06
0.08
0.10
0.15
0.20
0.30
0.35
0.40
0.42
0.44
0.46
0.47
0.48
0.49
0.51
0.52
0.53
0.54
0.56
0.58
0.60
0.65
0.70
0.80
0.90
0.95
0.97
0.98
0.99

$\Psi_{d}(z)$

31.7769 16.7611

0.7734

7.7294

6.8449

6.3933

5.9537

.8597

.9792

6.1460

6.4698

.7077

.1010

7.8841
8.6660

10.2294

14.9195

$-3.8451$

0.8439

2.4053

3.9599

4.3425

4.5665

4.8424

4.9436

4.9082

4.5655

4.1367

3.8355

3.6292

3.3684
$-68.7877$

$-34.4093$

$-22.9567$

-17.2357
-11.5253

$-8.6813$

$-6.9842$

$-4.7523$

$-2.7475$

$-2.7475$

$-2.6160$

$-2.9365$

$-3.3144$

$-4.1440$

$-5.0064$

$-6.7607$

$-12.0788$

9. 3445

4. 0231

2.2632

1.3929

0.5405

0.1301

$-0.1051$

$-0.3927$

$-0.516 C$

$-0.6185$

-0.670 T

$-0.6961$

$-0.7072$

$-0.7135$

$-0.7196$
$\Psi_{\mathrm{dU}}(\mathrm{z})$

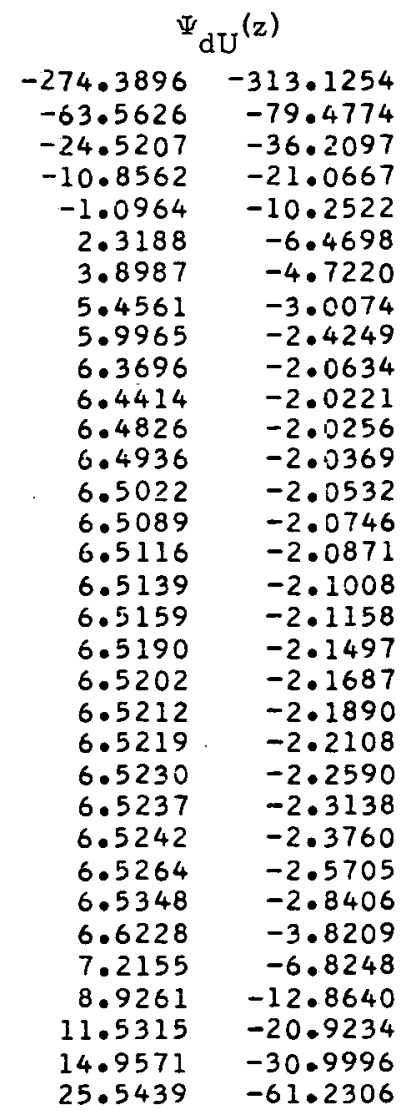

$\frac{\mathrm{z}}{\mathrm{h}}=1.000$

$\Psi_{\mathrm{dD}}(\mathrm{z})$

$5.3103-3.1644$

$5.3102-3.1642$

$5.3101-3.1637$

$\begin{array}{ll}5.3098 & -3.1631 \\ 5.3091 & -3.1614\end{array}$

$5.3081-3.15 .39$

$5.3068 \quad-3.1558$.

$5.3021-3.1453$

$5.2949-3.1311$

$\begin{array}{ll}5.2711 & -3.0948 \\ 5.2529 & -3.0747\end{array}$

$\begin{array}{ll}5.2529 & -3.0747 \\ 5.2291 & -3.0553\end{array}$

$\begin{array}{ll}5.2291 & -3.0553 \\ 5.2177 & -3.0482\end{array}$

$5.2051-3.0417$

$5.1912 \quad-3.0360$

$5.1838 .-3.0335$

$5.1760-3.0313$

$5.1678 \quad-3.0294$

$5.1502-3.0266$

$5.1407-3.0258$

$5.1308 \quad-3.0255$

$5.1204-3.0256$

$5.0982 \quad-3.0277$

$5.0738-3.0323$

$5.0471-3.0401$

$4.9685-3.0778$

$4.8696-3.1536$

$4.5829-3.5440$

$4.0769-5.0715$

$3.6403 \quad-8.4025$

$3.4073-12.5297$

$3.2941-18.6162$

$-35.7121$

$\begin{array}{cc}\Psi_{\mathrm{dI}}^{\prime}(\mathrm{z}) & \Psi_{\mathrm{dUI}}^{\prime}(\mathrm{z}) \\ 2.1601 & 0.3090 \\ 2.1584 & 0.3137 \\ 2.1556 & 0.3214 \\ 2.1517 & 0.3322 \\ 2.1403 & 0.3632 \\ 2.1245 & 0.4065 \\ 2.1041 & 0.4622 \\ 2.0332 & 0.6556 \\ 1.9335 & 0.9262 \\ 1.6457 & 1.7011 \\ 1.4556 & 2.2082 \\ 1.2322 & 2.7994 \\ 1.1329 & 3.0607 \\ 1.0276 & 3.3370 \\ 0.9158 & 3.6291 \\ 0.8574 & 3.7813 \\ 0.7973 & 3.9378 \\ 0.7353 & 4.0987 \\ 0.6058 & 4.4345 \\ 0.5380 & 4.6097 \\ 0.4681 & 4.7901 \\ 0.3960 & 4.9759 \\ 0.2448 & 5.3647 \\ 0.0834 & 5.7785 \\ -0.0894 & 6.2205 \\ -0.5818 & 7.4751 \\ -1.1906 & 9.0207 \\ -3.0798 & 13.8242 \\ -8.0542 & 26.6312 \\ -17.4725 & 51.1394 \\ -29.8677 & 83.5080 \\ -45.3105 & 123.8762 \\ -91.5711 & 244.8620 \\ & \end{array}$


$\Omega=15.00$

$k_{0} z$
0.00
0.10
0.20
0.30
0.40
0.50
0.60
0.70
0.80
0.90
0.95
0.97
0.98
0.99

0.00

0.10
0.20

0.20
0.30

0.40

0.50

0.60

0.70

0.80

0.90

0.95

0.97

0.98

0.99
$\Psi_{d}(z)$

$\begin{array}{ll}11.8977 & -0.0800 \\ 12.1996 & -0.0862 \\ 12.3141 & -0.0923 \\ 12.3593 & -0.0983 \\ 12.3539 & -0.1042 \\ 12.3006 & -0.1100 \\ 12.1926 & -0.1159 \\ 12.0100 & -0.1217 \\ 11.7025 & -0.1276 \\ 11.1046 & -0.1336 \\ 10.4654 & -0.1366 \\ 9.9860 & -0.1378 \\ 9.6069 & -0.1384 \\ 8.9751 & -0.1390\end{array}$

12.6444
12.6356
12.6087
12.5617
12.4988
12.3895
12.2460
12.0375
11.7120
11.1030
10.4605
9.9802
9.6008
8.9687

-0.6331
-0.6338
-0.6358
-0.6392
-0.6441
-0.6504
-0.6583
-0.6680
-0.6795
-0.6931
-0.7008
-0.7040
-0.7057
-0.7074

13.7817
12.7135
12.3362
12.1227
11.9642
11.8150
11.6459
11.4252
11.0964
10.4905
9.8516
9.3731
8.9946
8.3636
$\Psi_{\mathrm{dU}}(\mathrm{z})$

$\begin{array}{ll}12.2367 & -0.0996 \\ 12.2269 & -0.0996 \\ 12.1966 & -0.0997 \\ 12.1440 & -0.0998 \\ 12.0652 & -0.0999 \\ 11.9536 & -0.1001 \\ 11.7971 & -0.1004 \\ 11.5729 & -0.1007 \\ 11.2287 & -0.1010 \\ 10.5977 & -0.1014 \\ 9.9430 & -0.1016 \\ 9.4575 & -0.1017 \\ 9.0754 & -0.1017 \\ 8.4406 & -0.1018\end{array}$

$$
\Omega=15.00
$$

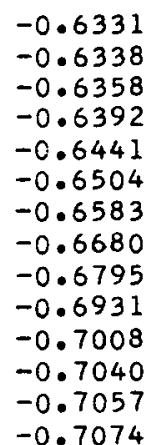

-0.7057
-0.7074

$\Omega=15.00$ $\frac{\mathrm{z}}{\mathrm{h}}=0.125$

$$
\Psi_{\mathrm{dD}}(\mathrm{z})
$$

$12.2313-0.1004$ 12.2209

12.1890

12.1336

12.0509
11.9343

11.7718

11.5404

11.1879

10.5476

9.8877

9.4001

9.0169
8.3811

$-0.1003$

$-0.1002$

$-0.1001$

$-0.0999$

$-0.0997$

$-0.0995$

$-0.0992$

$-0.0989$

$-0.0987$

$-0.0987$

-0.0986
-0.0986

$$
\frac{\mathrm{z}}{\mathrm{h}}=0.250
$$

12.6227
12.6109
12.5746
12.5120
12.4193
12.2898
12.1116
11.8619
11.4883
10.8243
10.1517
9.6588
9.2730
8.6344

$-0.6520$

$-0.6515$

$-0.6501$

$-0.6476$

$-0.6443$

$-0.6400$

$-0.6347$

$-0.6286$

$-0.6286$

$-0.6216$

$-0.6137$

$-0.6095$

$-0.6077$

$-0.6069$

$-0.6060$

$$
\frac{\mathrm{z}}{\mathrm{h}}=0.375
$$

-1.4821
-1.4870
-1.5018
-1.5272
-1.5645
-1.6154
-1.6829
-1.7710
-1.8861
-2.0380
-2.1323
-2.1742
-2.1962
-2.2189
$\Psi_{\mathrm{dI}}^{\mathrm{i}}(\mathrm{z}) \quad \Psi_{\mathrm{dUI}}^{\prime}(\mathrm{z})$

$-0.7432 \quad-0.3832$

$-0.7431 \quad-0.3831$

$-0.7427-0.3829$

$-0.7420 \quad-0.3826$

$-0.7410 \quad-0.3821$

$\begin{array}{ll}-0.7398 & -0.3815 \\ -0.7384 & -0.3807\end{array}$

$-0.7366 \quad-0.3798$

$-0.7346 \quad-0.3788$

$-0.7323 \quad-0.3776$

$-0.7311-0.3770$

$-0.7306 \quad-0.3767$

$-0.7303 \quad-0.3766$

$-0.3764$

$\begin{array}{ll}-2.1616 & -2.1616 \\ -2.1600 & -2.1600 \\ -2.1551 & -2.1551 \\ -2.1471 & -2.1471 \\ -2.1359 & -2.1359 \\ -2.1216 & -2.1216 \\ -2.1042 & -2.1042 \\ -2.0838 & -2.0838 \\ -2.0605 & -2.0605 \\ -2.0344 & -2.0344 \\ -2.0203 & -2.0203 \\ -2.0145 & -2.0145 \\ -2.0115 & -2.0115 \\ -2.0086 & -2.0086\end{array}$

$-2.0086-2.0086$

$\begin{array}{ll}-2.6764 & -4.0986 \\ -2.6720 & -4.0914 \\ -2.6587 & -4.0700 \\ -2.6368 & -4.0345 \\ -2.6064 & -3.9853 \\ -2.5678 & -3.9227 \\ -2.5212 & -3.8473 \\ -2.4672 & -3.7598 \\ -2.4061 & -3.6609 \\ -2.3384 & -3.5514 \\ -2.3024 & -3.4931 \\ -2.2875 & -3.4691 \\ -2.2800 & -3.4570 \\ -2.2725 & -3.4447\end{array}$


$\Psi_{d}(z)$

$\mathrm{k}_{\mathrm{o}} \mathrm{z}$

0.01

0.02

0.03

0.04

0.06

0.08

0.15

0.20

0.30

0.40

0.50

0.60

0.70

0.80

0.90

0.95

0.97

0.98

0.99

27.6144
17.0477
13.8551
12.4149
11.1830
10.7103
10.5067
10.3909
10.4397
13.5986
10.7192
10.7790
10.7721
10.6825
10.4643
9.9560
9.3630
8.9024
8.5329
7.9108

$-49$

$-49.5677$

$-24.7858$

$-16.5260$

$-12.3967$

$-8.2688$

$-6.2061$

$-4.9695$

$-2.5042$

$-1.6916$

$-1.2927$

$-1.0600$

$-0.9114$

$-0.8117$

$-0.7439$

$-0.6990$

$-0.6834$

$-0.6784$

$-0.6761$

$-0.6740$

12.9439
13.0365
13.4819
14.1935
$\vdots 5.3001$
16.0175
17.1705
19.3193
24.7044
62.4537
-13.0721
2.0220
5.7886
7.4954
8.4666
9.5258
10.5526
10.9266
11.1648
11.1443
10.9470
10.4487
9.8620
9.4048
9.0372
8.4171

$-3.4330$

$-3.6991$

$-4.9431$

$-6.8785$

$-9.8479$

$-11.7626$

$-14.832 \mathrm{~g}$

$-20.5435$

$-34.8349$

$-134.9305$

55.2867

25.2530

15.2503

10.7076

8.1146

.2678

2.4362

1.3144

0.3414

$-0.1055$

$-0.3774$

$-0.5795$

$-0.6701$

$-0.7056$

$-0.7234$

0.98

0.99
$\Omega=15.00$

${ }^{I} \mathrm{dU}^{(\mathrm{z})}$

$12.5731 \quad-2.2252$

12.5731

12.5731

$-2.2258$

$-2.2268$

$-2.2282$

$-2.2322$

$-2.2378$

$-2.2451$

$-2.2708$

$-2.3079$

$-2.4218$

$-2.6039$

$-2.8879$

$-3.3431$

$-4.1339$

$-5.7549$

$-10.6839$

$-20.5846$

$-33.7974$

$-50.3172$

$-99.8813$

$\Omega=15.00$

12.9439

13.0365

13.4819

14.1935

15.3001

16.0175

17.1705

17.1705

24.7044

62.4537

$-13.0721$

2.0220

5.7886

7.4954

8.4666

.5258

10.5526

10.9266

11.1648

11.1443

10.9470

10.4487

9.8620

9.4043

9.0372

8.4171

$-3.4330$

$-3.6991$

$-4.9431$

$-6.8785$

$-9.8479$

$-11.7626$

$-14.8328$

$-20.5435$

$-34.8349$

$-134.9306$

65.2867

25.2530

15.2503

10.7076

8.1146

5.2678
2.4362

1.3144

0.3414

$-0.1055$

$-0.3774$

$-0.5796$

$-0.6701$

$-0.7056$

$-0.7234$ $\frac{z}{h}=0.500$

$\Psi_{\mathrm{dD}}(\mathrm{z})$

$12.2075 \quad-2.3982$

$12.2071-2.3980$

$12.2064 \quad-2.3977$

$12.2053-2.3972$

$12.2024 \quad-2.3957$

$12.1983-2.3937$

$12.1930-2.3911$

$12.1746 \quad-2.3822$

12.1485

$12.072 ?$

11.9606

11.8070

11.6000

11.3168

10.9055

10.2000

9.5052

9.0032

8.6128
7.9696

$-2.3698$

2.3345

$-2.2245$

$-2.1513$

$-2.0673$

$-1.9737$

1.8184

$-1.7965$

$-1.7855$

$-1.7745$

$\frac{\mathrm{z}}{\mathrm{h}}=0.750$

11.0305

11.0237

11.0023

10.9855

10.9732

10.9687
0.9640

10.9640

10.9591

10.9539
10.9486

10.9430

10.9371

10.9310

10.9247

10.9181

$10.90<0$

10.8636

10.8146

10.6826

10.4838

10.1650

9.5589

8.9165

8.4359

8.0563
7.4240

$-2.9863$

$-2.9476$

$-2.9190$

$-2.8990$

$-2.8845$

$-2.8769$

$-2.8691$

$-2.8610$

$-2.8528$

-2.8443
-2.8356

$-2.8267$

$-2.8176$

$-2.7987$

$-2.7481$

$-2.6929$

$-2.5703$

$-2.4348$

$-2.2911$

-2.144 ?

$-2.0725$

$-2.044$

-2.0300
-2.0160
$\Psi_{\mathrm{dI}}^{\prime}(\mathrm{z})$

$\Psi_{\mathrm{dUI}}^{\mathrm{t}}(\mathrm{z})$

$-1.5572$

$-1.5571$

$-1.5570$

$-1.5568$

$-1.5563$

$-1.5556$

$-1.5518$

$-1.5477$

$-1.5359$

$-1.5197$

$-1.4991$

$-1.4746$

$-1.4464$

$-1.4150$

$-1.3627$

$-1.3553$

-1.3516
-1.3479

$-4.4498$

-4.4486
-4.4475

$-4.4445$

$-4.4403$

$-4.4161$

$-4.3899$

$-4.3157$

$-4.2132$

$-4.0841$

$-3.9301$

$-3.5567$

$-3.3427$

$-3.2301$

$-3.1842$

-3.1610
-3.1377

2.0110

I. 9625

1.818

1.7109

1.6089

1.6089
1.5811

1. 5526

1. 5231

1.4928

1.4616

1.3968

1.3631

1. 3286

1. 2571

1.0646

0.8529

0.3761

$-0.1658$

$-0.7669$

$-1.4253$

$-1.7773$

$-1.9229$

$-1.9968$

$-2.0714$

$-2.0110$

$-1.9625$ 
$\Omega=15.00$

$\mathrm{k} z$
0.00
0.05
0.10
0.12
0.14
0.16
0.17
0.18
0.19
0.21
0.22
0.23
0.24
0.26
0.28
0.30
0.35
0.40
0.45
0.50
0.52
0.54
0.56
0.57
0.58
0.59
0.61
0.62
0.63
0.64
0.66
0.68
0.70
0.75
0.80
0.90
0.95
0.97
0.98
0.99

ko

0.00

0.10

0.12

0.16

0.18

.26

30

0.35

0.54

.56

0.57
0.58

.59

.61

0.64

.66

0.70

.90

.95

0.98
0.99
$\Psi_{d}(z)$

12.4378

13.8232

15.5517

18.5889

22.2582

25.8954

33.1420

54.8317

$-31.7967$

$-10.1113$

0.7550

4. 3945

4.3945
6.2246

7. 3288

8.8122

9.5565

9.9971

10.2793

10.3630

10.4338

10.4934

10.5193

10.5430

10.5644

10.6009

10.6162

10.6296

10.6411

10.6586

10.6691

10.6724

10.6487

10.5724

10.1540

9.6011

9.1563

8.7946

8.1803
$-0.8746$

$-1.1002$

-1.5412
-1.8685

$-2.4113$

$-3.4930$

$-4.5728$

$-6.7303$

$-13.1987$

2.6629
6.1945

6.1945
4.0369

2.9571

1.8752

1.3322

1.0046

0.5626
0.3357

0.1945

0.0957

0.0635

0.0344

0.0078

0.0047

0.0168

$-0.0284$

$-0.0609$

$-0.0712$

$-0.0811$

0.1002

$-0.1184$

$-0.1359$

$-0.2164$

$-0.2923$

$-0.3310$

$-0.3469$

-0.3550
-0.3632
$\mathrm{dU}^{(\mathrm{z})}$

\begin{tabular}{ll}
\multicolumn{2}{c}{$\Psi_{\mathrm{dU}}(\mathrm{z})$} \\
12.4983 & -2.7622 \\
12.5013 & -2.7788 \\
12.5109 & -2.8300 \\
12.5168 & -2.8611 \\
12.5241 & -2.8989 \\
12.5329 & -2.9440 \\
12.5380 & -2.9694 \\
12.5435 & -2.9970 \\
12.5495 & -3.0267 \\
12.5632 & -3.0935 \\
12.5710 & -3.1308 \\
12.5795 & -3.1709 \\
12.5887 & -3.2141 \\
12.6097 & -3.3106 \\
12.6347 & -3.4224 \\
12.6644 & -3.5525 \\
12.7681 & -3.9841 \\
12.9405 & -4.6558 \\
13.2544 & -5.8024 \\
13.9301 & -8.1300 \\
14.4564 & -9.8863 \\
15.3506 & -12.8207 \\
17.1664 & -18.6996 \\
18.9970 & -24.5834 \\
22.6740 & -36.3555 \\
33.7382 & -71.6805 \\
-10.6241 & 69.6450 \\
0.4383 & 34.3197 \\
4.1123 & 22.5471 \\
5.9388 & 16.6626 \\
7.7425 & 10.7817 \\
8.6194 & 7.8445 \\
9.1232 & 6.0846 \\
9.7161 & 3.7443 \\
9.9000 & 2.5797 \\
.9 .6578 & 1.4224 \\
9.1209 & 1.0934 \\
8.6751 & 0.9867 \\
8.3117 & 0.9376 \\
7.6949 & 0.8910
\end{tabular}

$\frac{\mathrm{h}}{\mathrm{z}}=0.625$

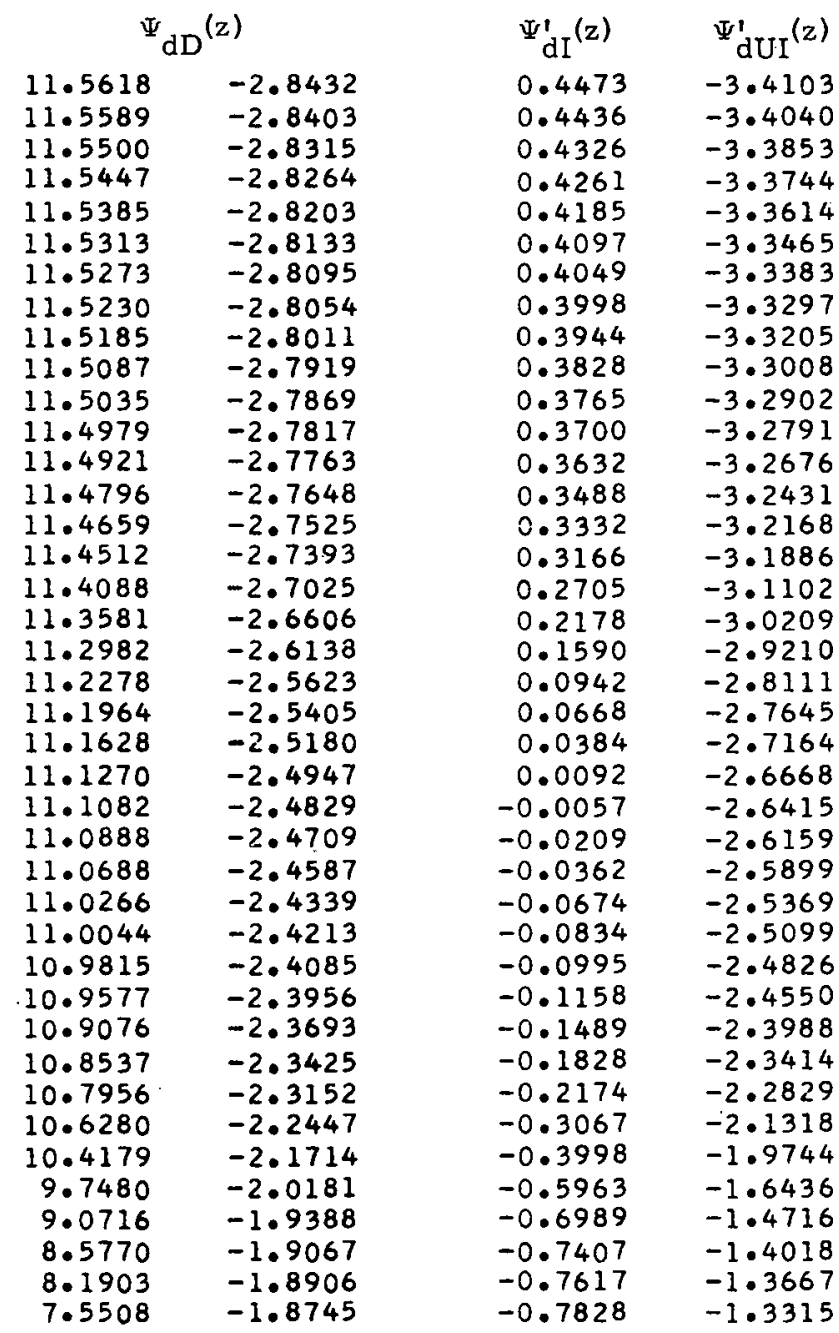


$\Omega=15.00$

$$
\Psi_{d}(z)
$$

$\mathrm{k}_{\mathrm{o}} \mathrm{z}$

0.00

0.10

0.15
0.20

0.22

0.24

0.25

0.26

0.27

0.28

0.29

0.30

0.31

0.32

0.34

0.36

0.38

0.39

0.40

0.41
0.42

0.43

0.44

0.45

0.46

0.48

0.50
0.55

0.65

0.70

0.70

0.80
0.90

0.90
0.95

0.97

0.98

0.99
$14.0551 \quad-6.7833$

$11.9466 \quad-4.6862$

$11.6319 \quad-4.2706$

$11.4239-4.0808$

$1.3549 \quad-4.0633$

$11.2893-4.0826$

$11.2569-4.1079$

$11.2244 \quad-4.1451$

$11.1913-4.1959$

11.1572

11.1215

11.0835

11.0425

10.9973

10.8875

10.7313

10.4691

10.2461

9.8756
9.1192

6.6260

38.8723

14.6669

13.0422

12.4438

11.9310

11.6914

11.3988

11.2310

10.9599

10.6323

10.0542

9.4382

8.9707

8.5981

$-4.1959$

$-4.2621$

$-4.3466$

$-4.4530$

$-4.5861$

$-4.7527$

$-5.2291$

$-6.0302$

$-7.5491$

$-8.9240$

$-11.2819$

$-16.2088$

$-32.6923$

181.9370

21.0054

10.2970

6.4161

3.2042

1.8103

0.3762

$-0.188 \equiv$

$-0.6535$

$-0.854 \mathrm{C}$

$-0.979]$

$-1.034 \mathrm{C}$

-1.056 :

$-1.0675$
15.2050

18.3334

(a)

$-19.8842$

8.5264

9.5415

10.3306

10.4 .988

10.6383

10.7556

10.8552

10.9406

11.0143

11.0784

11.1836

11.2653

11.3295

11.3564

11.3803

11.4016

11.4206

11.4374

11.4524

11.4656

11.4772

11.4963

11.5103

11.5275

11.5235

11.4587

11.2995

10.9199

10.4377

10.0349

9.6978

$\Psi_{\mathrm{dU}}^{(z)}$ $-4.58$

$-7.8003$

31.3597

2.2183

1. 1682

0.5596

0.3430

0.0152

$-0.1116$

$-0.2205$

$-0.3152$

$-0.3982$

$-0.4716$

$-0.5958$

$-0.7821$

-0.7821
-0.8197

$-0.8546$

$-0.8872$

$-0.9179$

$-0.9467$

$-0.9740$

$-1.0000$

$-1.0247$

$-1.0713$

$-1.1147$

$-1.2141$

$-1.3074$

$-1.7376$

$-2.0770$

$-2.3179$

$-2.4350$

-2.4992
-2.5677

$\frac{z}{h}=0.875$

$\Psi_{\mathrm{dD}}(\mathrm{z})$

$\Psi_{\mathrm{dI}}^{\prime}(\mathrm{z})$

$\Psi_{\mathrm{dUI}}^{\prime}(\mathrm{z})$

2.4931

$-0.6977$

$\begin{array}{ll}10.6692 & -3.0766 \\ 10.6657 & -3.0659\end{array}$

2.4156

2.3191

2.1847

2.1204

$-0.6018$

$10.6610 \quad-3.0526$

$10.6506-3.0256$

$10.6467 \quad-3.0162$

$10.6445-3.0112$

$10.6422-3.0061$

10.6398

10.6373

10.6345

10.6317

10.6287

10.6255

10.6186

10.6110

10.6025

10.5025

10.5932

10.5932

10.5829

10.5774

10.5716

10.5656

0.559

10.5305

10.4863

10.4305

10.2700

9.9993

9.4607

8.8661

8.4090

8.0423
7.4236

$-3.0007$

$-2.9952$

$-2.9837$

$-2.9778$

$-2.9716$

$-2.9589$

$-2.9456$

$-2.9317$

$-2.9246$

$-2.9174$

2.9101

$-2.8951$

$-2.8874$

$-2.8797$

$-2.8719$

$-2.8561$

$-2.8401$

2.79

$-2.6834$

$-2.6305$

$-2.6292$

$-2.6656$

$-2.6910$

$-2.7067$

$-2.7246$

2.0502

1.9741

1.9338

1.8922

1.8490

1.8045

1.7584

1.7110

1.6119

1.3968

1.3395

1. 2809

1.2209

1. 1595

1.0967

1.0326

0.9671

0.9002

0.7624

0.6191

0.2370

$-0.1802$

$-1.1283$

$-2.2641$

$-3.6920$

$-4.5902$

$-5.002$

-5.2227
-5.4540

$-0.3161$

$-0.2365$

$-0.1497$

$-0.1035$

$-0.0555$

0.0459

0.0993

0.1545

0.2114

0.2702

0.3929

0.5227

0.6595

0.7304

0.8031

0.8776

0.9537

1.0316

1.1112

1. 1925

1.2755

1.4466

1.6247

2.1003

2.6209

.8110

7.1001

8.2822

8.8297

9.1237 
$\Omega=15.00$

$k{ }^{z}$
0.01
0.02
0.03
0.04
0.06
0.08
0.10
0.15
0.20
0.30
0.35
0.40
0.42
0.44
0.46
0.47
0.48
0.49
0.51
0.52
0.53
0.54
0.56
0.58
0.60
0.65
0.70
0.80
0.90
0.95
0.97
0.98
0.99

$\Psi_{d}(z)$

$\begin{array}{lr}38.9524 & -68.8798 \\ 22.7098 & -34.4554 \\ 17.6229 & -22.9875 \\ 15.2343 & -17.2588 \\ 13.0527 & -11.5409 \\ 12.1051 & -8.6930 \\ 11.6182 & -6.9937 \\ 11.1359 & -4.7589 \\ 11.0254 & -3.6829 \\ 11.1435 & -2.7518 \\ 11.3249 & -2.6204 \\ 11.6859 & -2.7493 \\ 11.9538 & -2.9423 \\ 12.3986 & -3.3213 \\ 13.2863 & -4.1535 \\ 14.1739 & -5.0183 \\ 15.9493 & -6.7777 \\ 21.2773 & -12.1112 \\ -0.0440 & 9.3736 \\ 5.2828 & 4.0368 \\ 7.0564 & 2.2718 \\ 7.9412 & 1.3991 \\ 8.8213 & 0.5441 \\ 9.2552 & 0.1325 \\ 9.5093 & -0.1034 \\ 9.8226 & -0.3919 \\ 9.9389 & -0.515 .7 \\ 9.9037 & -0.6186 \\ 9.5118 & -0.6710 \\ 8.9728 & -0.6965 \\ 8.5347 & -0.7078 \\ 8.1767 & -0.7138 \\ 7.5663 & -0.7201\end{array}$

$\Psi_{\mathrm{dU}}(\mathrm{z})$

$-265.8990-314.4396$ $-57.6292 \quad-79.8067$ $-19.0608 \quad-36.3567$ $-5.5621-21.1499$ $4.0793-10.2897$ $4.0793-10.2897$ $\begin{array}{ll}7.4531 & -6.4913 \\ 9.0139 & -4.7361\end{array}$ 10.5523 11.0 .862 11.4547

11.5256

11.5664 11.5772 11.5857

11.5924

11.5950

11.5974

11.5994

11.6025

11.6037

11.6047

11.6055

11.6067

11.6076

11.6082

11.6110

11.6205

11.7136

11.7136

12.3309

14.1205

1.6 .8758

20.5377

32.0218
$-3.0143$

$-2.4292$

$-2.0660$

$-2.0244$

$-2.0277$

$-2.0389$

$-2.0552$

$-2.0765$

$-2.0890$

$-2.1028$

$-2.1178$

$-2.1517$

$-2.1707$

$-2.1910$

$-2.2128$

$-2.2610$

$-2.3159$

$-2.3781$

$-2.5728$

$-2.8431$

$-3.8243$

$-12.8765$

$-20.9441$

$-31.0305$

$-61.2922$

$$
\frac{\mathrm{z}}{\mathrm{h}}=1.000
$$

$$
\Psi_{\mathrm{dD}}(\mathrm{z})
$$

10.4017

10.4016

10.4015
10.4012

10.4005

10.3995

10.3995
10.3982

10.3935

10.3864
10.3627

10.3445

10.3208

10.3095

10.2969

10.2831

10.2757

10.2680

10.2598

10.2423

10.2329

10.2230

10.2127

10.1906

10.1664

10.1399

10.0619

9.9640

9.6822

9.2001

8.8422

8.7590

8.8823

9.7643
$-3.1693$

$-3.1691$

$-3.1686$

$-3.1663$

$-3.1663$

$-3.1638$

-3.1607
-3.1502

$-3.1502$

$-3.1360$

$-3.0998$

$-3.0798$

$-3.0605$

$-3.0534$

$-3.0470$

$-3.0413$

$-3.0388$

$-3.0366$

$-3.0347$

$-3.0320$

$-3.0312$

$-3.0309$

$-3.0311$

$-3.0332$

$-3.0380$

$-3.0459$

$-3.0839$

$-3.1601$

$-3.5522$

$-5.0851$

$-8.4271$

$-12.9691$

$-18.6740$

$-35.8255$

\begin{tabular}{lr}
$\Psi_{\mathrm{dI}}^{\prime}(z)$ & \multicolumn{1}{c}{$\Psi_{\mathrm{dUI}}^{\prime}(z)$} \\
2.1630 & 0.3103 \\
2.1613 & 0.3150 \\
2.1585 & 0.3227 \\
2.1545 & 0.3335 \\
2.1432 & 0.3645 \\
2.1274 & 0.4079 \\
2.1069 & 0.4636 \\
2.0360 & 0.6571 \\
1.9362 & 0.9279 \\
1.6483 & 1.7033 \\
1.4580 & 2.2107 \\
1.2345 & 2.8022 \\
1.1352 & 3.0637 \\
1.0297 & 3.3403 \\
0.9179 & 3.6326 \\
0.8595 & 3.7849 \\
0.7993 & 3.9415 \\
0.7373 & 4.1025 \\
0.6077 & 4.4386 \\
0.5398 & 4.6139 \\
0.4699 & 4.7944 \\
0.3978 & 4.9803 \\
0.2465 & 5.3694 \\
0.0850 & 5.7836 \\
-0.0880 & 6.2259 \\
-0.5807 & 7.4815 \\
-1.1898 & 9.0285 \\
-3.0803 & 13.8364 \\
-8.0579 & 26.6561 \\
17.4822 & 51.1889 \\
29.8853 & 83.5904 \\
45.3379 & 123.9995 \\
91.6278 & 245.1082
\end{tabular}



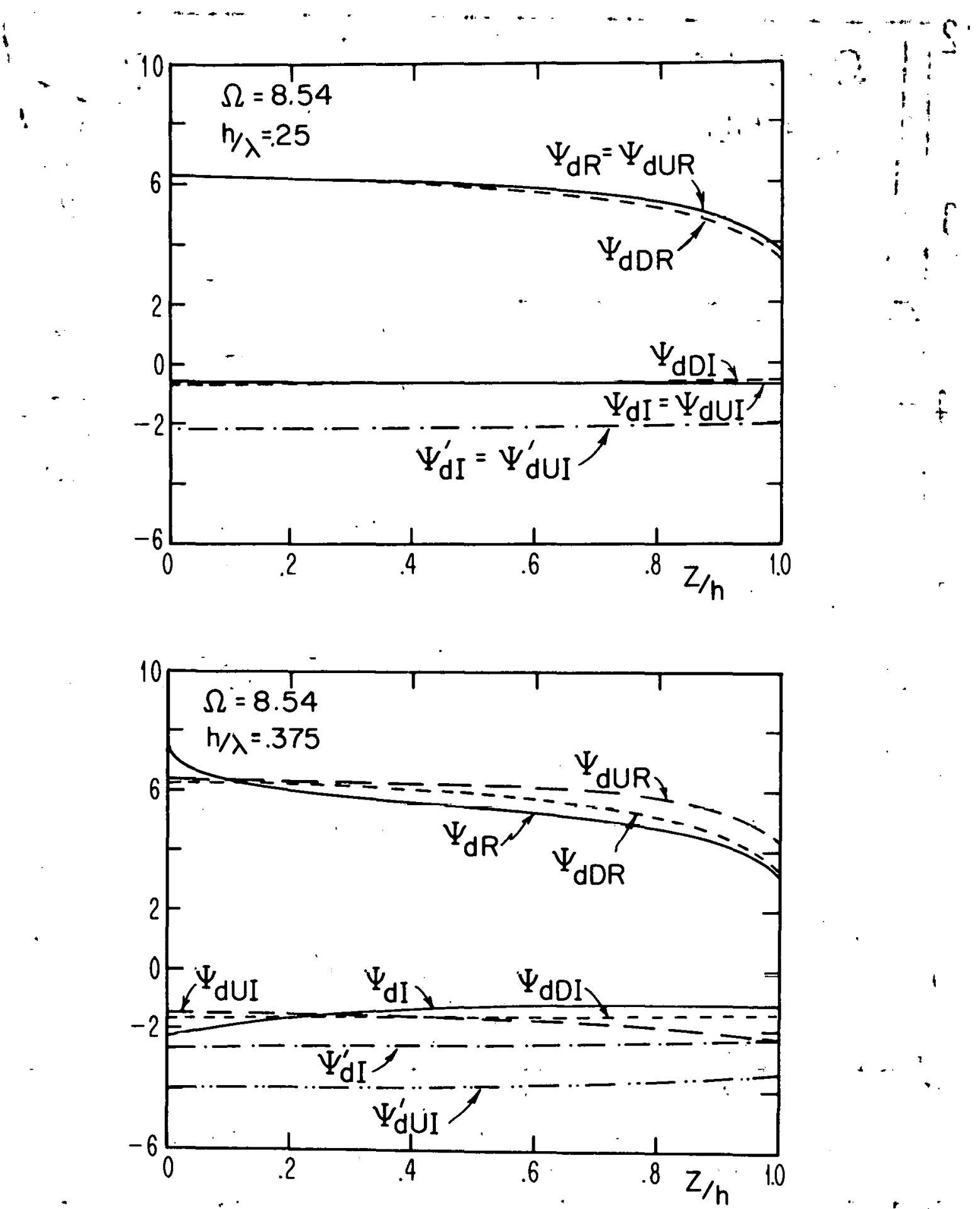

FIG. 1 THE FUNCTIONS $\Psi_{d R}, \Psi_{d I}, \Psi_{d U R}$,

$\therefore \quad \Psi_{d U I}, \Psi_{d D R}, \Psi_{d D I}, \Psi_{d I}^{\prime}, \Psi_{d U I}^{\prime}$ FOR $h / \lambda=.25$ AND .375; $\Omega=8.54$. 


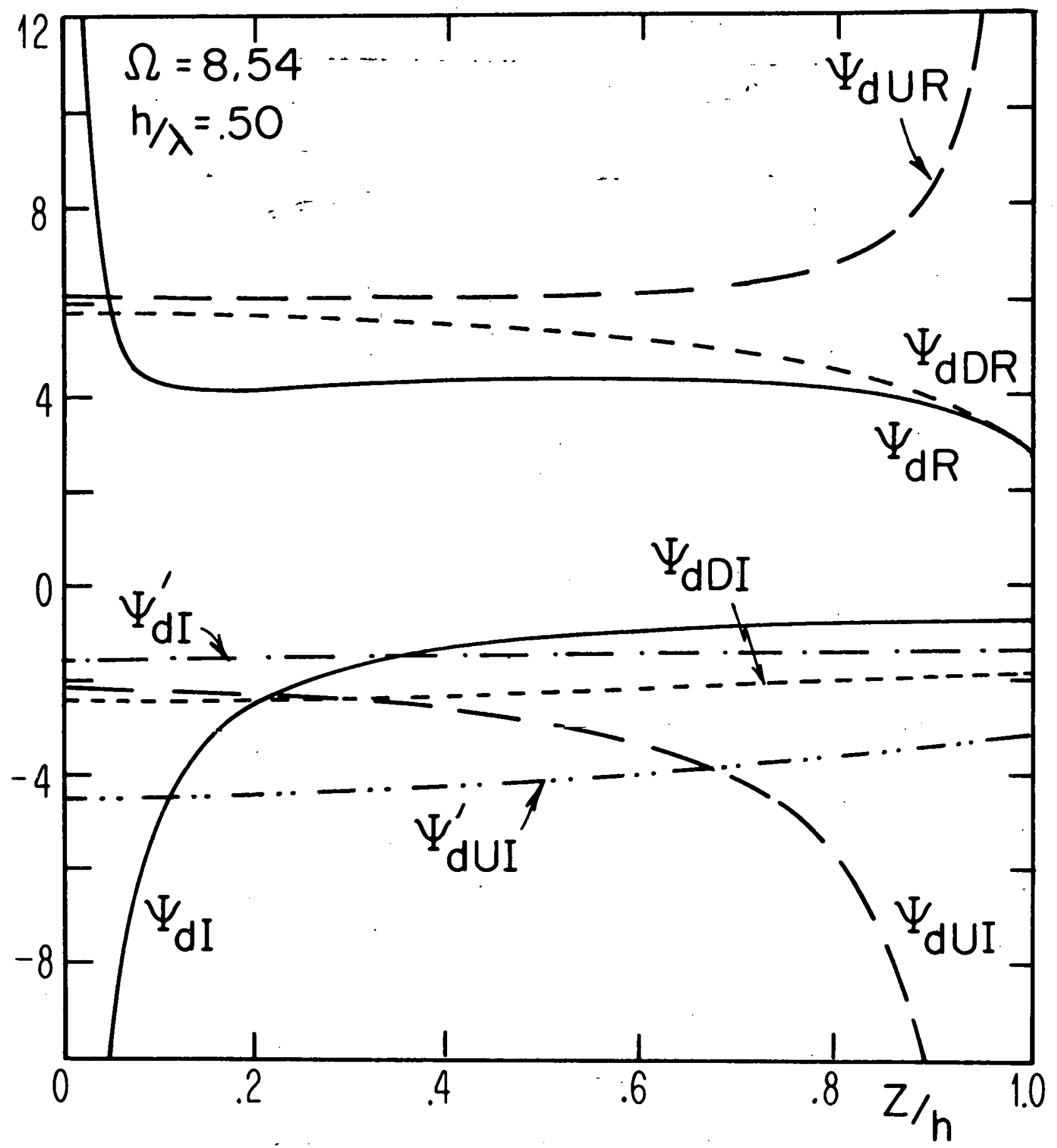

FIG. 2 THE FUNCTIONS $\Psi_{\mathrm{dR}}, \Psi_{\mathrm{dI}}, \Psi_{\mathrm{dUR}}$, $\Psi_{d U I}, \Psi_{d D R}, \Psi_{d D I}, \Psi_{d I}^{\prime}, \Psi_{d U I}^{\prime}$, FOR $h / \lambda=.50 ; \Omega=8.54$. 


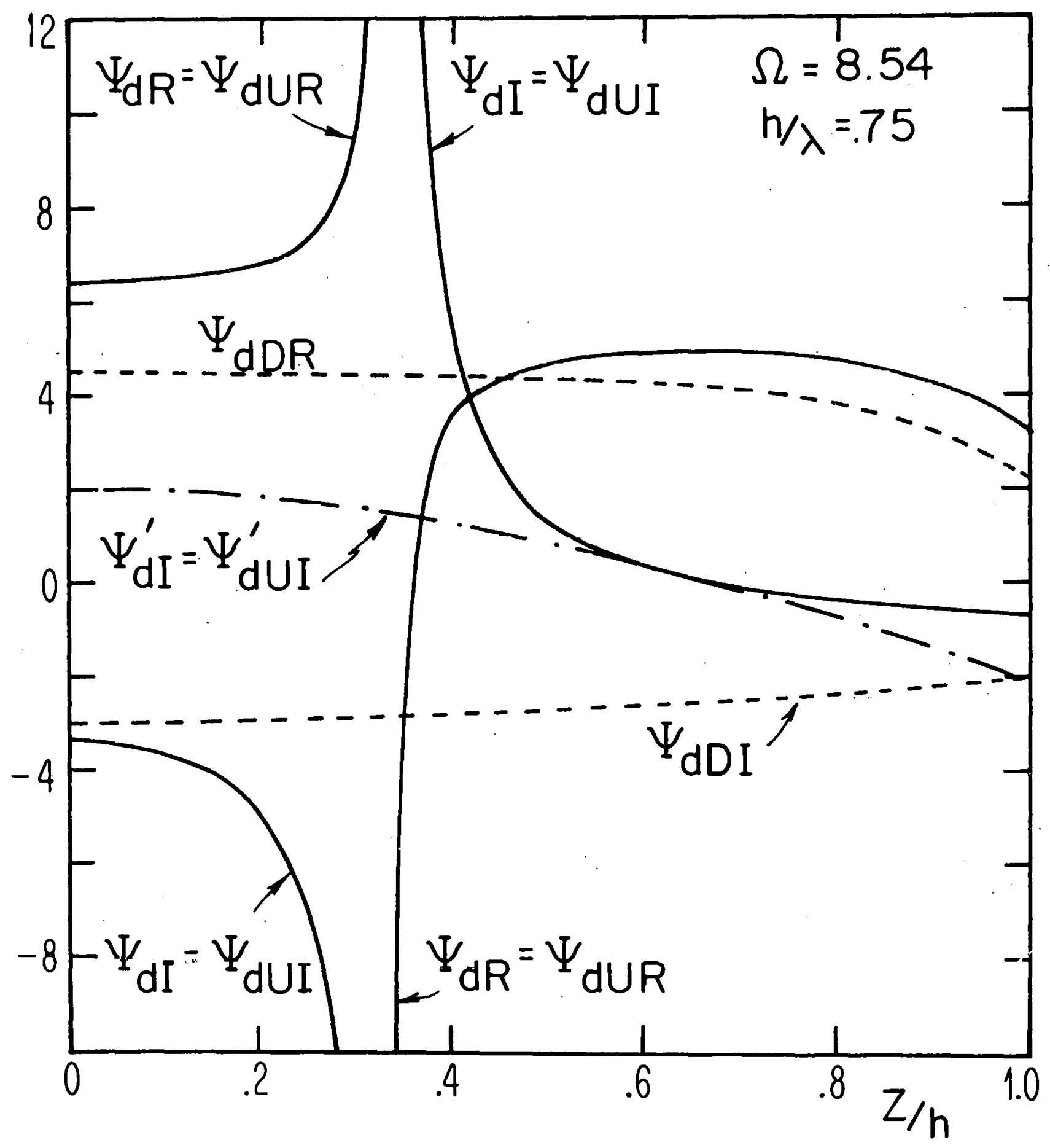

FIG. 3 THE FUNCTIONS $\Psi_{\mathrm{dR}}, \Psi_{\mathrm{dI}}, \Psi_{\mathrm{dUR}}$, $\Psi_{\mathrm{dUI}}, \Psi_{\mathrm{dDR}}, \Psi_{\mathrm{dDI}}, \Psi_{\mathrm{dI}}^{\prime}, \Psi_{\mathrm{dUI}}^{\prime}$, FOR $h / \lambda=.75 ; \Omega=8.54$. 


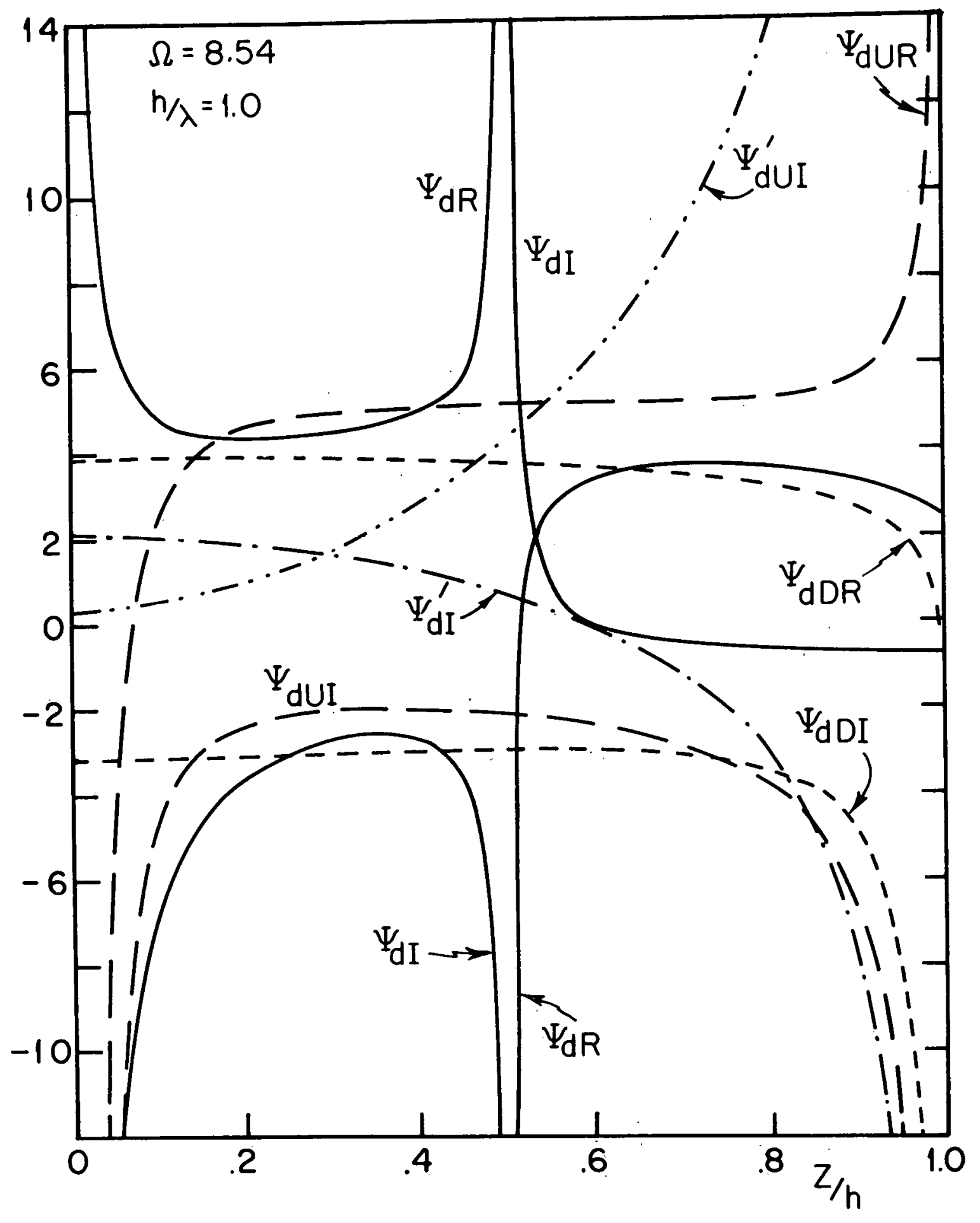

FIG. 4 THE FUNCTIONS $\Psi_{\mathrm{dR}}, \Psi_{\mathrm{dI}}, \Psi_{\mathrm{dUR}}$, $\Psi_{\mathrm{dUI}}, \Psi_{\mathrm{dDR}}, \Psi_{\mathrm{dDI}}, \Psi_{\mathrm{dI}}^{\prime}, \Psi_{\mathrm{dUI}}^{\prime}$, FOR $\mathrm{h} / \lambda=1.0 ; \Omega=8.54$. 